\title{
Growth Performance, Meat Quality and Antioxidant Status of Sheep Supplemented with Tannins: A Meta-Analysis
}

\author{
José Felipe Orzuna-Orzuna ${ }^{1}\left(\mathbb{D}\right.$, Griselda Dorantes-Iturbide ${ }^{1}\left(\mathbb{D}\right.$, Alejandro Lara-Bueno ${ }^{1, *} \mathbb{*}$, \\ Germán David Mendoza-Martínez ${ }^{2}{ }^{\oplus}$, Luis Alberto Miranda-Romero ${ }^{1}$ and Héctor Aarón Lee-Rangel ${ }^{3}$ \\ 1 Departamento de Zootecnia, Universidad Autónoma Chapingo, Chapingo CP 56230, Mexico; \\ jforzuna@gmail.com (J.F.O.-O.); griseldi0993@gmail.com (G.D.-I.); \\ albertomiranda@correo.chapingo.mx (L.A.M.-R.) \\ 2 Unidad Xochimilco, Departamento de Producción Agrícola y Animal, Universidad Autónoma Metropolitana, \\ Mexico City CP 04960, Mexico; gmendoza@correo.xoc.uam.mx \\ 3 Centro de Biociencias, Facultad de Agronomía y Veterinaria, Instituto de Investigaciones en Zonas Desérticas, \\ Universidad Autónoma de San Luis Potosí, San Luis Potosí CP 78321, Mexico; hector.lee@uaslp.mx \\ * Correspondence: alarab_11@hotmail.com
}

check for

updates

Citation: Orzuna-Orzuna, J.F.; Dorantes-Iturbide, G.; Lara-Bueno, A.; Mendoza-Martínez, G.D.;

Miranda-Romero, L.A.; Lee-Rangel, H.A. Growth Performance, Meat Quality and Antioxidant Status of Sheep Supplemented with Tannins: A Meta-Analysis. Animals 2021, 11, 3184 https://doi.org/10.3390/ani11113184

Academic Editors: Susana Chamorro and Carlos Romero

Received: 5 October 2021

Accepted: 5 November 2021

Published: 8 November 2021

Publisher's Note: MDPI stays neutral with regard to jurisdictional claims in published maps and institutional affiliations.

Copyright: (c) 2021 by the authors. Licensee MDPI, Basel, Switzerland. This article is an open access article distributed under the terms and conditions of the Creative Commons Attribution (CC BY) license (https:// creativecommons.org/licenses/by/ $4.0 /)$.
Simple Summary: Tannins can be used to improve productive performance, meat quality and antioxidant status of ruminants. The objective of this study was to evaluate the effects of dietary tannin supplementation on productive performance, carcass characteristics, meat quality and blood serum antioxidant status of sheep through a meta-analysis. Only studies with weaned or older sheep were included. The sheep included in the present study were between 2 and 6 months old, and between 12 and $31 \mathrm{~kg}$ of body weight. Tannin supplementation improved productive performance, carcass yield, meat oxidative stability and blood serum antioxidant capacity. This suggests that the inclusion of tannins in sheep diets could be used to improve growth and reduce oxidative stress in animals, and to improve meat quality and shelf life.

Abstract: The objective of this study was to evaluate the effects of dietary supplementation with tannins (TANs) on productive performance, carcass characteristics, meat quality, oxidative stability, and blood serum antioxidant capacity of sheep through a meta-analysis. Using Scopus, Web of Science, ScienceDirect, and PubMed databases, a systematic search was performed for studies published in scientific journals that investigated the effects of TANs supplementation on the variables of interest. Only studies with weaned or older sheep were included. The data analyzed were extracted from 53 peer-reviewed publications. The sheep included in the present study were between 2 and 6 months old, and between 12 and $31 \mathrm{~kg}$ of body weight. The effects of TANs were analyzed using random-effects statistical models to examine the standardized mean difference (SMD) between treatments with TANs and control (no TANs). Heterogeneity was explored by meta-regression and a subgroup analysis was performed for covariates that were significant. Supplementation with TANs did not affect dry matter intake, $\mathrm{pH}$, color $\left(\mathrm{L}^{*}\right.$ and $\left.\mathrm{b}^{*}\right)$, Warner-Bratzler shear force, cooking loss and meat chemical composition $(p>0.05)$. Supplementation with TANs increased daily weight gain $(\mathrm{SMD}=0.274, p<0.05)$, total antioxidant capacity $(\mathrm{SMD}=1.120, p<0.001)$, glutathione peroxidase enzyme activity $(\mathrm{SMD}=0.801, p<0.001)$ and catalase (SMD $=0.848, p<0.001)$, and decreased malondialdehyde (MDA) concentration in blood serum (SMD $=-0.535, p<0.05)$. Supplementation with TANs decreased feed conversion rate (SMD $=-0.246, p<0.05)$, and the concentration of MDA $(\mathrm{SMD}=-2.020, p<0.001)$ and metmyoglobin (SMD $=-0.482, p<0.05)$ in meat. However, meat redness $(\mathrm{SMD}=0.365)$, hot carcass yield $(\mathrm{SMD}=0.234)$, cold carcass yield $(\mathrm{SMD}=0.510)$, backfat thickness (SMD = 0.565) and the Longissimus dorsi muscle area (SMD $=0.413$ ) increased in response to TANs supplementation $(p<0.05)$. In conclusion, the addition of tannins in sheep diets improves productive performance, antioxidant status in blood serum, oxidative stability of meat and some other characteristics related to meat and carcass quality.

Keywords: oxidative stability; natural antioxidants; polyphenolic compounds; meta-regression 


\section{Introduction}

Antibiotics (e.g., monensin) have been used for several decades as growth promoters in animals [1]. However, the inappropriate use of these products results in an accumulation of toxic residues in meat, which can affect the health of the consumer [2,3]. In addition, the emergence of bacterial strains resistant to the antibiotic effects [4] as well as the prohibition of these compounds in some countries [5] have led the industry and researchers to search for alternative products with similar effects as antibiotics, but of natural origin. Tannins (TANs), which are derived from plants, have received special attention and are among the most studied bioactive compounds, particularly in ruminants [1]. TANs are a group of polyphenolic compounds present in a wide variety of plants, which can be grouped into hydrolysable tannins (HTs) and condensed tannins (CTs) based on their chemical structure [6,7]. TANs can produce positive effects in animals, such as those of the antioxidant, antimicrobial, antiparasitic, immunomodulatory, and anti-inflammatory varities [1,6].

TANs can play an important role in the nutritional value of the feed, the quality of the products obtained, and the health and welfare of the animals [8]. Dietary inclusion of TANs at low to moderate concentrations ( 20 to $45 \mathrm{~g} \mathrm{~kg}^{-1} \mathrm{DM}$ ) can improve growth rate and feed utilization efficiency in ruminants, mainly due to a reduction in protein degradation in the rumen and a subsequent increase in the flow of amino acids to the small intestine [9-11]. However, large amounts ( $\left.>55 \mathrm{~g} \mathrm{~kg}^{-1} \mathrm{DM}\right)$ of TANs in the diet reduce feed intake, rumen microbial activity, nutrient digestibility and endogenous digestive enzyme activity $[1,9,10,12]$, resulting in lower feed efficiency and growth rate.

Particularly in sheep, several studies have been conducted to evaluate the effects of dietary supplementation with extracts of TANs and TANs-rich plants on productive performance $[13,14]$, carcass characteristics [15,16], oxidative stability and physicochemical characteristics of meat [17-20], and blood serum antioxidant status [21,22]. However, the results are still not consistent, probably as a consequence of variability among the studies regarding feeding conditions, age of the animals, type of product used, dosage and source of TANs $[1,8]$. Therefore, identifying the factors that contribute to this variability is a key aspect in the development of products containing TANs that can be used to improve productive performance, meat and carcass quality, and antioxidant status of sheep.

Some review articles $[1,8,23,24]$ have suggested that dietary supplementation with TANs can improve productive performance, meat quality and antioxidant status of livestock. However, these reviews did not use a meta-analysis approach and also did not focus only on sheep. In addition, narrative reviews can lead to biased conclusions because they lack a methodological approach and are subjective to the author's interpretation of previous research [25]. In contrast, meta-analysis (MA) is a statistical tool that allows synthesizing data published in different studies in a quantitative way [26,27]. Furthermore, MA allows us to explore the heterogeneity sources among the diverse studies, which helps to obtain additional information about the factors contributing to the variability of the observed outcomes in response to a specific treatment [28]. MA has been frequently used in biomedical and clinical research, but its use in research related to secondary plant metabolites and meat science is still limited [29]. The objective of this meta-analysis was to evaluate the effect of dietary supplementation with tannins on productive performance, carcass characteristics, meat quality and oxidative stability, and antioxidant status of sheep blood plasma. The heterogeneity of responses was also examined using meta-regression analysis with the purpose of identifying factors contributing to the variability in the response variables.

\section{Materials and Methods}

\subsection{Literature Search and Study Selection}

To perform a robust meta-analysis, the Preferred Reporting Items for Systematic Reviews and Meta-Analyses (PRISMA) guidelines [30] were used in the identification, selection, choice and inclusion of information, as shown in Figure S1. To identify studies that evaluated the effect of supplementation with TANs on productive performance, carcass and meat quality characteristics, and antioxidant status of sheep blood serum, a systematic 
literature search was performed in the scientific databases of Scopus, ScienceDirect, Web of Science, and PubMed. The following keywords were used in all databases: tannin, lamb, sheep, growth performance, intake, carcass characteristics, meat quality, antioxidant status, and oxidative stability. The search and selection process was limited to the results of papers published between January 2010 and June 2021, where 1157 scientific publications were identified (Figure S1). These publications went through a two-step selection process as previously described by other authors [31,32]. First, a selection of titles and abstracts was performed excluding simulation studies, review articles, studies not conducted in sheep, invitro studies, and articles that did not include the variables of interest. Subsequently, to be considered, studies had to meet inclusion criteria previously used by other authors [31-33]: (1) Use of sheep and specify the procedure used to randomly assign animals within treatments; (2) data on productive performance, oxidative stability of meat, meat and/or carcass quality characteristics, or blood serum antioxidant status; (3) similarity between control and experimental groups except for the presence of TANs; (4) quantification or possible determination of the amount of TANs in the diet; (5) peer-reviewed journal articles written in English; (6) least squares means of the control and experimental groups with measures of variability (standard deviation or standard error); and (7) sample size.

\subsection{Data Extraction}

After exclusion of duplicate papers and selection of titles and abstracts, 99 full-text articles were evaluated; of these, only 53 articles met the inclusion criteria (Table A1) and were used to obtain the quantitative data for the meta-analysis. To be considered, variables had to be reported in at least three studies [32,34]. Consequently, the response variables included in the meta-analysis were: daily weight gain, dry matter intake, feed conversion rate (feed intake/weight gain), hot and cold carcass yield, backfat thickness, Longissimus dorsi muscle area, meat quality characteristics ( $\mathrm{pH}$, color, chemical composition, malondialdehyde content, among others), as well as total antioxidant capacity, malondialdehyde content (as an indicator of lipid oxidation) and antioxidant enzyme activity (superoxide dismutase, catalase and glutathione peroxidase) in blood serum. In addition, when available, additional data were collected, such as: characteristics of the published study (author and year of publication), chemical composition of the diet, amount of forage in the diet $\left(\mathrm{g} \mathrm{kg}^{-1} \mathrm{DM}\right)$, number of replicates, amount of TANs in the $\operatorname{diet}\left(\mathrm{g} \mathrm{kg}^{-1} \mathrm{DM}\right)$, period of supplementation with TANs (days), type of TANs (HTs, CTs or mixture of both), source of botanical origin of the TANs, and method of inclusion of the TANs (extract or naturally present in the diet). The references of the articles included in the dataset are listed in Table A1. Averages, standard deviation (SD), and number of replicates for each treatment were extracted from these articles. When the articles presented the SD of each experimental group, these values were used directly in the meta-analysis. Where the SD was not reported, it was calculated by multiplying the standard error of the means (SEM) by the square root of the sample size, using the equation: $\mathrm{SD}=\mathrm{SEM} \times \sqrt{ } \mathrm{n}$, as previously reported by Higgins and Thomas [35], where $n=$ number of replicates.

\subsection{Calculations and Statistical Analysis}

Meta-analysis and meta-regression data were analyzed using the Open Meta-analyst for Ecology and Evolution software [36]. Response variables were analyzed using the standardized mean difference (SMD), also referred to as effect size (ES), in which the difference between the means of the experimental and control groups was standardized using the SD of the groups with and without TANs [37]. SMDs were calculated using methods previously described by DerSimonian and Laird [38] for random-effects models. The SMD is a more robust estimate of ES when heterogeneity exists in the data set [39]. The variables of the chemical composition of the diets were analyzed with the MEANS procedure using the SAS statistical program [40] to obtain descriptive statistics values. Differences in the composition of the diets of the control and TANs-supplemented treatments were evaluated 
by the MIXED procedure, using the studies as random effect and Tukey's test to detect differences between treatments, as previously reported by other authors [32,41].

\subsection{Heterogeneity}

Heterogeneity was measured using the $\mathrm{I}^{2}$ statistic and the chi-square $(\mathrm{Q})$ test [42]. Because of the relatively low capacity of the $\mathrm{Q}$ test to detect heterogeneity among a small number of treatment comparisons, an $\alpha$ level of 0.10 was used [39,43]. $I^{2}$ (percentage of variation) values range from 0 to $100 \%$, where values close to $25 \%$ indicate low heterogeneity, close to $50 \%$ indicate moderate heterogeneity, and close to $75 \%$ indicate high heterogeneity between studies [26,28]. Likewise, $\mathrm{I}^{2}$ values greater than $50 \%$ indicate significant heterogeneity [33].

\subsection{Publication Bias}

Since a visual inspection of funnel plots (generally used to assess publication bias) is subjective and must be balanced with additional analyses [44], three methods were used to assess evidence of publication bias: (1) the funnel plot [45]; (2) Begg's adjusted rank correlation [46]; and (3) Egger's regression asymmetry test [47]. Bias was considered to be present when the funnel plot showed asymmetry, or when at least one of the statistical methods (Begg's test and Egger's test) was significant $(p<0.10)$. Tests to assess publication bias are inappropriate when the variable to be assessed is not reported in at least 10 studies, and when significant heterogeneity $(\mathrm{Q})$ is detected with an $\alpha \leq 0.10$, because it may lead to false-positive claims [48]. Consequently, funnel plots, Begg's test, and Egger's test were only performed for variables that met the aforementioned criteria. In cases where statistical evidence of publication bias was found, the "trim-and-fill" method of Duval and Tweedie was used to estimate the number of possible missing observations [49].

\subsection{Meta-Regression}

Sources of parameter heterogeneity that showed Q with an $\alpha$ level of $\leq 0.10$ [43] or $\mathrm{I}^{2}$ greater than $50 \%$ [26] were assessed by meta-regression analysis. Similar to the publication bias tests, meta regression analysis was only performed for response variables that were reported in at least 10 studies [44]. The meta regression was estimated using the DerSimonian and Laird method of moments, which is well known for estimating variance among studies [26]. Continuous and categorical variables were used in the meta-regression. The continuous variables included: differences in neutral detergent fiber (NDF) and ether extract (EE) content in the diets $\left(\mathrm{g} \mathrm{kg}^{-1} \mathrm{DM}\right)$, TANs dose $\left(\mathrm{g} \mathrm{kg}^{-1} \mathrm{DM}\right)$, and duration of the experimental phase (days). Categorical variables included: source of botanical origin of TANs, method by which TANs were supplied (extract or as part of some dietary ingredient), type of TANs (CTs, HTs or mixture of both), and age of animals ( $\leq 3$ months of age or $>3$ months of age). When categorical covariates were significant at an $\alpha$ level of $\leq 0.05$, SMD was assessed by subgroup analysis [32,41]. Likewise, when the meta regression was significant $(p \leq 0.05)$ for supplementation level and experimental period, these covariates were evaluated by subgroup analysis by dividing the covariates as follows: dietary TANs supplementation level ( $\leq 20$ or $\left.>20 \mathrm{~g} \mathrm{~kg}^{-1} \mathrm{DM}\right)$; and experimental period ( $\leq 70$ or $>70$ days).

\section{Results}

\subsection{Study Attributes and Excluded Studies}

Descriptive statistics and mean test for diet composition are presented in Table 1. Except for NDF, EE and organic matter (OM) content, no significant differences were observed between the control and TANs treatment for the rest of the nutritional components of the diet. Among the nutritional components, only fiber and fat content seem to have considerable effects on productive performance, carcass characteristics and meat quality [50]. Thus, it is possible to exclude the effects of the rest of the diet components on the response of animals to TANs supplementation for the data set. 
Table 1. Descriptive statistics of the complete data set for the effect of tannins supplementation to sheep diets.

\begin{tabular}{|c|c|c|c|c|c|c|c|c|c|c|c|}
\hline \multicolumn{2}{|l|}{ Parameter } & \multicolumn{2}{|c|}{ Mean } & \multicolumn{2}{|c|}{ Median } & \multicolumn{2}{|c|}{ Minimum } & \multicolumn{2}{|c|}{ Maximum } & \multicolumn{2}{|c|}{ SD } \\
\hline Dietary Features & NC & Control & Tannin & Control & Tannin & Control & Tannin & Control & Tannin & Control & Tannin \\
\hline Forage, $\mathrm{g} \mathrm{kg}^{-1} \mathrm{DM}$ & 122 & 428.5 & 432.2 & 400.0 & 400.0 & 0 & 0 & 1000 & 1000 & 265.6 & 261.3 \\
\hline $\mathrm{DM}, \mathrm{g} \mathrm{kg}^{-1}$ & 100 & 874.8 & 871.7 & 900.0 & 903.4 & 160.0 & 156.0 & 953.9 & 947.5 & 122.8 & 130.8 \\
\hline $\mathrm{OM}, \mathrm{g} \mathrm{kg}^{-1} \mathrm{DM}$ & 46 & $857.5^{b}$ & $866.8^{a}$ & 910.0 & 922.0 & 148.0 & 146.0 & 957.2 & 984.5 & 193.7 & 188.7 \\
\hline $\mathrm{CP}, \mathrm{g} \mathrm{kg}^{-1} \mathrm{DM}$ & 124 & 156.3 & 156.9 & 157.0 & 156.5 & 84.0 & 89.0 & 251.0 & 255.0 & 26.9 & 26.5 \\
\hline $\mathrm{EE}, \mathrm{g} \mathrm{kg}^{-1} \mathrm{DM}$ & 106 & $35.5^{b}$ & $40.7^{\mathrm{a}}$ & 29.3 & 36.3 & 13.0 & 12.8 & 81.0 & 98.3 & 16.6 & 18.3 \\
\hline $\mathrm{NDF}, \mathrm{g} \mathrm{kg}^{-1} \mathrm{DM}$ & 122 & $388.8^{\mathrm{a}}$ & $375.9^{b}$ & 385.7 & 365.5 & 156.0 & 152.0 & 731.1 & 704.9 & 179.6 & 108.7 \\
\hline $\mathrm{ADF}, \mathrm{g} \mathrm{kg}^{-1} \mathrm{DM}$ & 94 & 211.2 & 212.1 & 190.0 & 179.5 & 81.0 & 69.8 & 516.0 & 496.5 & 94.1 & 94.2 \\
\hline $\mathrm{Ca}, \mathrm{g} \mathrm{kg}^{-1} \mathrm{DM}$ & 24 & 7.0 & 7.2 & 6.1 & 6.7 & 3.4 & 3.6 & 17.0 & 18.0 & 3.1 & 3.4 \\
\hline $\mathrm{P}, \mathrm{g} \mathrm{kg}^{-1} \mathrm{DM}$ & 22 & 4.0 & 4.0 & 4.4 & 4.4 & 2.0 & 2.0 & 5.8 & 6.2 & 1.2 & 1.3 \\
\hline $\mathrm{ME}, \mathrm{MJ} \mathrm{kg}{ }^{-1} \mathrm{DM}$ & 56 & 10.6 & 10.6 & 10.5 & 10.7 & 9.2 & 8.8 & 12.5 & 12.6 & 0.8 & 0.9 \\
\hline Tannin, $\mathrm{g} \mathrm{kg}^{-1} \mathrm{DM}$ & 135 & - & 20.2 & - & 15.5 & - & 0.02 & - & 132.0 & - & 20.6 \\
\hline Duration, days & 135 & \multicolumn{2}{|c|}{70.0} & \multicolumn{2}{|c|}{70.0} & \multicolumn{2}{|c|}{28.0} & \multicolumn{2}{|c|}{180.0} & \multicolumn{2}{|c|}{30.0} \\
\hline
\end{tabular}

NC: number of comparisons; SD: standard deviation; DM: dry matter; OM: organic matter; CP: crude protein; EE: ether extract; NDF: neutral detergent fiber; ADF: acid detergent fiber; Ca: calcium; P: phosphorus; MJ: megajoule; ${ }^{\text {a }},{ }^{\text {b }}$ in the same row, means followed by different letters differ significantly by the Tukey test $(p<0.05)$.

The studies included in the present meta-analysis were conducted in 21 different countries, predominantly in Brazil (17\%) and Iran (13.2\%). The experimental doses of TANs ranged from 0.02 to $132 \mathrm{~g} \mathrm{~kg}^{-1} \mathrm{DM}$, and the duration of the experimental periods varied from 28 to 180 days (Table 1). The TANs used were divided into: CTs, HTs and mixture of both. Of the treatments, $54.7 \%$ used mixtures of CTs and HTs, $39.6 \%$ used CTs and the remaining $5.7 \%$ used HTs. Moreover, $79.3 \%$ of the treatments used plant parts, forages or by-products containing TANs in natural form, and $20.7 \%$ used extracts of TANs in the diets. The studies included in the meta-analysis used a total of 36 different sources of TANs, the majority of treatments (13.2\%) used TANs from Vitis vinifera, 9.4\% from Cistus ladanifer, $7.5 \%$ from plant mixtures and $9.4 \%$ from pomegranate (Punica granatum) and in the other $60.5 \%$ of the treatments, 32 other different sources of TANs were used.

\subsection{Growth Performance and Carcass Characteristics}

Table 2 shows that dietary supplementation with TANs increased $(p<0.05)$ daily weight gain (DWG), hot carcass yield (HCY), cold carcass yield (CCY), backfat thickness (BFT) and Longissimus dorsi muscle area (LMA). There was no observed a significant impact of the inclusion of TANs in the diet on dry matter intake (DMI; $p>0.05$ ). In addition, feed conversion ratio (FCR) decreased in response to dietary supplementation with TANs $(p<0.05)$.

Table 2. Growth performance and carcass characteristics of sheep supplemented with tannins.

\begin{tabular}{|c|c|c|c|c|c|c|c|c|c|c|}
\hline \multirow[b]{2}{*}{ Parameter } & \multirow[b]{2}{*}{$\mathbf{N}$} & \multirow[b]{2}{*}{ NC } & \multirow[b]{2}{*}{ SMD } & \multicolumn{4}{|c|}{$95 \%$ CI } & \multicolumn{3}{|c|}{ Heterogeneity } \\
\hline & & & & SE & Lower & Upper & $p$-Value & $\mathbf{Q}$ & $p$-Value & $I^{2}(\%)$ \\
\hline Daily weight gain (DWG) & 42 & 104 & 0.274 & 0.116 & 0.046 & 0.501 & 0.018 & 472.57 & $<0.001$ & 78.20 \\
\hline Dry matter intake (DMI) & 42 & 104 & 0.090 & 0.124 & -0.152 & 0.333 & 0.466 & 524.508 & $<0.001$ & 80.36 \\
\hline Feed conversion ratio (FCR) & 27 & 60 & -0.308 & 0.127 & -0.556 & -0.060 & 0.015 & 197.05 & $<0.001$ & 70.06 \\
\hline Hot carcass yield (HCY) & 26 & 59 & 0.234 & 0.108 & 0.023 & 0.445 & 0.030 & 142.03 & $<0.001$ & 59.16 \\
\hline Cold carcass yield (CCY) & 9 & 23 & 0.510 & 0.228 & 0.063 & 0.957 & 0.025 & 86.09 & $<0.001$ & 74.45 \\
\hline Backfat thickness (BFT) & 9 & 24 & 0.565 & 0.193 & 0.188 & 0.943 & 0.003 & 77.94 & $<0.001$ & 70.49 \\
\hline Longissimus dorsi muscle area (LMA) & 10 & 22 & 0.413 & 0.170 & 0.080 & 0.747 & 0.015 & 52.40 & $<0.001$ & 59.92 \\
\hline
\end{tabular}

N: number of studies; NC: number of comparisons; SMD: standardized mean difference; CI: confidence interval of SMD; SE: standard error. Q: chi-squared statistic and associated significance level ( $p$-value); ${ }^{2}$ : percentage of variation.

\subsection{Meat Quality Characteristics}

No significant effects of TANs inclusion in the sheep diet $(p>0.05)$ were observed on meat $\mathrm{pH}$, meat lightness $\left(\mathrm{L}^{*}\right)$ and yellowness $\left(\mathrm{b}^{*}\right)$, Warner-Bratzler shear force (WBSF), meat cooking loss (CL), protein content, intramuscular fat (IMF) and meat ash (Table 3). Dietary supplementation with TANs decreased drip loss (DL) and meat moisture $(p<0.05)$. While meat redness $\left(a^{*}\right)$ increased in response to dietary supplementation with TANs 
$(p<0.05)$. In addition, malondialdehyde content in raw meat (MDAc) and metmyoglobin $(\mathrm{Met} \mathrm{Mb})$ content of meat decreased in response to dietary supplementation with TANs $(p<0.05)$.

Table 3. Meat characteristics of sheep supplemented with tannins.

\begin{tabular}{|c|c|c|c|c|c|c|c|c|c|c|}
\hline \multirow[b]{2}{*}{ Parameter } & \multirow[b]{2}{*}{$\mathbf{N}$} & \multirow[b]{2}{*}{ NC } & \multirow[b]{2}{*}{ SMD } & \multicolumn{4}{|c|}{$95 \% \mathrm{CI}$} & \multicolumn{3}{|c|}{ Heterogeneity } \\
\hline & & & & SE & Lower & Upper & $p$-Value & Q & $p$-Value & $I^{2}(\%)$ \\
\hline Meat $\mathrm{pH}$ & 19 & 52 & 0.037 & 0.098 & -0.156 & 0.230 & 0.706 & 89.29 & $<0.001$ & 42.88 \\
\hline Lightness $\left(\mathrm{L}^{*}\right)$ & 20 & 54 & 0.008 & 0.128 & -0.243 & 0.260 & 0.950 & 151.88 & $<0.001$ & 65.10 \\
\hline Redness $\left(a^{*}\right)$ & 20 & 54 & 0.365 & 0.120 & 0.129 & 0.601 & 0.002 & 133.39 & $<0.001$ & 62.27 \\
\hline Yellowness $\left(b^{*}\right)$ & 20 & 54 & 0.048 & 0.145 & -0.236 & 0.332 & 0.742 & 186.70 & $<0.001$ & 71.61 \\
\hline WBSF & 15 & 42 & -0.027 & 0.093 & -0.210 & 0.155 & 0.769 & 53.74 & 0.088 & 23.71 \\
\hline Drip loss (DL) & 4 & 17 & -2.828 & 0.516 & -3.839 & -1.817 & $<0.001$ & 149.57 & $<0.001$ & 89.30 \\
\hline Cooking loss (CL) & 14 & 42 & 0.105 & 0.216 & -0.317 & 0.528 & 0.626 & 243.00 & $<0.001$ & 83.13 \\
\hline Moisture & 5 & 16 & -0.693 & 0.333 & -1.345 & -0.041 & 0.037 & 77.25 & $<0.001$ & 80.58 \\
\hline Protein & 8 & 23 & 0.249 & 0.282 & -0.304 & 0.802 & 0.378 & 114.45 & $<0.001$ & 80.78 \\
\hline Intramuscular fat (IMF) & 16 & 40 & -0.168 & 0.186 & -0.532 & 0.196 & 0.366 & 172.04 & $<0.001$ & 77.33 \\
\hline Ash & 6 & 20 & 0.507 & 0.332 & -0.144 & 1.158 & 0.127 & 108.41 & $<0.001$ & 82.47 \\
\hline Malondialdehyde (MDAc) & 10 & 29 & -2.020 & 0.326 & -2.659 & -1.380 & $<0.001$ & 195.96 & $<0.001$ & 85.65 \\
\hline Metmyoglobin (MetMb) & 3 & 6 & -0.482 & 0.222 & -0.916 & -0.047 & 0.030 & 5.25 & 0.387 & 4.68 \\
\hline
\end{tabular}

N: number of studies; NC: number of comparisons; SMD: standardized mean difference; CI: confidence interval of SMD; SE: standard error. Q: chi-squared statistic and associated significance level ( $p$-value); $\mathrm{I}^{2}$ : percentage of variation; WBSF: Warner-Bratzler shear force.

\subsection{Antioxidant Status}

Table 4 shows that dietary supplementation with TANs increased total antioxidant capacity (TAC), and catalase (CAT) and glutathione peroxidase (GPx) enzyme activity in blood serum $(p<0.05)$. On the other hand, the concentration of malondialdehyde in blood serum (MDAs) decreased ( $p<0.05$; Table 2$)$ in response to TANs' supplementation. Moreover, no significant impact was observed for blood serum superoxide dismutase (SOD) enzyme activity $(p>0.05)$.

Table 4. Oxidative status of lambs supplemented with tannins.

\begin{tabular}{|c|c|c|c|c|c|c|c|c|c|c|}
\hline \multirow[b]{2}{*}{ Parameter } & \multirow[b]{2}{*}{$\mathbf{N}$} & \multirow[b]{2}{*}{ NC } & \multirow[b]{2}{*}{ SMD } & \multicolumn{4}{|c|}{$95 \%$ CI } & \multicolumn{3}{|c|}{ Heterogeneity } \\
\hline & & & & SE & Lower & Upper & $p$-Value & $\mathbf{Q}$ & $p$-Value & $I^{2}(\%)$ \\
\hline Total antioxidant capacity (TAC) & 9 & 17 & 1.120 & 0.222 & 0.686 & 1.555 & $<0.001$ & 43.661 & $<0.001$ & 63.35 \\
\hline Superoxide dismutase (SOD) & 6 & 14 & -0.122 & 0.328 & -0.766 & 0.521 & 0.709 & 61.306 & $<0.001$ & 78.79 \\
\hline Catalase (CAT) & 5 & 12 & 0.848 & 0.239 & 0.380 & 1.315 & $<0.001$ & 22.963 & 0.018 & 52.10 \\
\hline Glutathione peroxidase (GPx) & 3 & 6 & 0.801 & 0.209 & 0.392 & 1.211 & $<0.001$ & 2.267 & 0.811 & 0 \\
\hline Malondialdehyde (MDAs) & 7 & 17 & -0.535 & 0.244 & -1.014 & -0.056 & 0.029 & 54.824 & $<0.001$ & 70.81 \\
\hline
\end{tabular}

N: number of studies; NC: number of comparisons; SMD: standardized mean difference; CI: confidence interval of SMD; SE: standard error. Q: chi-squared statistic and associated significance level ( $p$-value); $\mathrm{I}^{2}$ : percentage of variation.

\subsection{Analysis of Publication Bias}

DWG, DMI, FCR, HCY, LMA, meat $\mathrm{pH}$, meat color $\left(\mathrm{L}^{*}\right.$, $\mathrm{a}^{*}$ and $\left.\mathrm{b}^{*}\right)$, WBSF, CL, IMF and MDAc had significant heterogeneity (Q) with an $\alpha \leq 0.10$. Whereas CCY, BFT, DL, moisture, protein, ash, MetMb, TAC, SOD, CAT, GPx and MDAs were reported in less than 10 studies. Therefore, tests to assess publication bias were not performed for any variable, because under these conditions they may result in false positive claims [48].

\subsection{Meta-Regression}

Significant heterogeneity ( $\mathrm{Q} ; p<0.10$ ) was observed for DWG, DMI, FCR, HCY, LMA (Table 2$)$, meat $\mathrm{pH}$, meat color $\left(\mathrm{L}^{*}, \mathrm{a}^{*}\right.$ and $\left.\mathrm{b}^{*}\right)$, WBSF, $\mathrm{DL}, \mathrm{CL}$, moisture content, protein, IMF, meat ash, MDAc, MetMb (Table 3), TAC, SOD, CAT, and MDAs (Table 4). Since it is not advisable to use meta-regression when there are fewer than 10 studies that reported 
the response variable of interest [44], this analysis was only performed for the variables: DWG, DMI, FCR, HCY, LMA, meat $\mathrm{pH}, \mathrm{L}^{*}, \mathrm{a}^{*}, \mathrm{~b}^{*}, \mathrm{CL}, \mathrm{IMF}$, and MDAc.

Table 5 shows that the dose of TANs explained $(p<0.05) 0.54,3.02,8.84,14.48,1.26,9.56$, and $17.0 \%$ of the heterogeneity observed for DWG, DMI, FCR, HCY, CL, IMF, and MDAc, respectively. The period of TANs supplementation had a significant relationship with DWG, DMI, FCR, LMA, $\mathrm{a}^{*}$, and MDAc $(p<0.05)$; however, it only explained between 1.15 and $26.30 \%$ of the observed heterogeneity in these variables. Animal age was significantly related to FCR, LMA, $\mathrm{a}^{*}$, $\mathrm{b}^{*}$, IMF and MDAc, explaining 6.57, 66.18, 28.53, 2.80, 14.55 and $56.60 \%$ of the observed heterogeneity, respectively $(p<0.05)$. The type of TANs explained between 11.65 and $54.54 \%$ of the observed heterogeneity for FCR, meat $\mathrm{pH}$, $\mathrm{L}^{*}, \mathrm{~b}^{*}$ and MDAc $(p<0.01)$. The method of inclusion of TANs in the diet explained 1.17, 23.06 and 5.11 of the observed heterogeneity in DWG, meat $\mathrm{pH}$ and MDAc, respectively $(p<0.05)$. The botanical origin of TANs had a significant relationship with DMI, FCR, HCY, LMA, meat $\mathrm{pH}, \mathrm{L}^{*}, \mathrm{~b}^{*}, \mathrm{CL}$, IMF and MDAc, explaining between 1.12 and $100 \%$ of the observed heterogeneity in these variables $(p<0.01)$. A significant relationship $(p<0.001)$ was observed between $\mathrm{a}^{*}$ and MDAc with dietary ether extract content (EED), where the variation in EED explained 19.28 and $4.60 \%$ of the heterogeneity observed in $\mathrm{a}^{*}$ and MDAc, respectively. A significant relationship $(p<0.05)$ was observed between HCY and $a^{*}$ with the neutral detergent fiber content of the diets (NDFD), where variation in NDFD explained 6.27 and $21.86 \%$ of the heterogeneity observed in HCY and $\mathrm{a}^{*}$, respectively.

\subsection{Subgroup Analysis}

Table S1 shows that DWG increased when doses of TANs lower than $20 \mathrm{~g} \mathrm{~kg}^{-1} \mathrm{DM}$ were used (SDM $=0.485 ; p<0.001)$, but doses higher than $20 \mathrm{~g} \mathrm{~kg}^{-1} \mathrm{DM}$ did not affect DWG (SMD $=-0.282 ; p>0.05)$. Similarly, DMI increased when doses of TANs lower than $20 \mathrm{~g} \mathrm{~kg}^{-1} \mathrm{DM}$ were used (SDM $\left.=0.324 ; p<0.05\right)$, but doses higher than $20 \mathrm{~g} \mathrm{~kg}^{-1} \mathrm{DM}$ did not affect DMI (SMD $=-0.416 ; p>0.05)$. Additionally, animals from studies using doses lower than $20 \mathrm{~g} \mathrm{~kg}^{-1} \mathrm{DM}$ had lower FCR (SMD $\left.=-0.423 ; p<0.001\right)$, but no differences were observed with respect to FCR in animals from studies using doses higher than $20 \mathrm{~g} \mathrm{~kg}^{-1} \mathrm{DM}(\mathrm{SMD}=0.640 ; p>0.05)$. HCY was higher in animals supplemented with doses of TANs lower than $20 \mathrm{~g} \mathrm{~kg}^{-1} \mathrm{DM}(\mathrm{SMD}=0.276 ; p<0.05)$, whereas doses higher than $20 \mathrm{~g} \mathrm{~kg}^{-1} \mathrm{DM}$ did not modify HCY (SMD $=0.152 ; p>0.05$ ). CL increased when TANs doses lower than $20 \mathrm{~g} \mathrm{~kg}^{-1} \mathrm{DM}$ were used (SMD $\left.=0.501 ; p<0.05\right)$ but decreased with doses higher than $20 \mathrm{~g} \mathrm{~kg}^{-1} \mathrm{DM}(\mathrm{SMD}=-1.158 ; p<0.05)$. Low doses of TANs $\left(<20 \mathrm{~g} \mathrm{~kg}^{-1} \mathrm{DM}\right)$ did not affect IMF content (SMD $=0.207 ; p>0.05)$, but doses higher than $20 \mathrm{~g} \mathrm{~kg}^{-1} \mathrm{DM}$ reduced IMF content $(\mathrm{SMD}=-0.691 ; p<0.001)$. MDAc decreased regardless of the dose of TANs used $(p<0.01)$; however, the effect was greater when doses lower than $20 \mathrm{~g} \mathrm{~kg}^{-1} \mathrm{DM}$ were used (SMD $=-2.735)$ compared to doses higher than $20 \mathrm{~g} \mathrm{~kg}^{-1} \mathrm{DM}(\mathrm{SMD}=-1.058)$.

Table S2 shows that DWG increased in response to dietary supplementation with TANs regardless of the supplementation period $(p<0.05)$. However, the effect was greater when TANs were offered for more than 70 days (SMD $=0.515$ ) compared to periods up to 70 days $(\mathrm{SMD}=0.256)$. On the other hand, DMI increased when dietary supplementation with TANs lasted more than 70 days (SMD $=0.590 ; p<0.05$ ) but was not affected when TANs were offered for up to 70 days (SMD $=-0.142 ; p>0.05$ ). In contrast, FCR decreased when dietary supplementation with TANs lasted more than 70 days (SMD $=-0.549 ; p<0.05)$ but was not affected when TANs were offered for up to 70 days (SMD $=-0.197 ; p>0.05$ ). Additionally, a* of meat increased when dietary supplementation with TANs lasted more than 70 days (SMD $=0.981 ; p<0.001$ ) but was not affected when TANs were offered for up to 70 days $(\mathrm{SMD}=-0.029 ; p>0.05)$. Higher LMA was observed when supplementation periods longer than 70 days were used (SMD $=0.870 ; p<0.01)$, but when the period lasted less than 70 days no significant effects were observed in LMA (SMD $=0.063 ; p>0.05$ ). MDAc decreased in response to dietary supplementation with TANs regardless of the supplementation period used $(p<0.001)$. However, the effect was greater when TANs 
were offered for more than 70 days (SMD $=-2.706)$ compared to periods up to 70 days $(\mathrm{SMD}=-0.784)$.

Table 5. Meta-regression of the effects of dietary tannins supplementation on growth performance, meat quality and antioxidant status of sheep.

\begin{tabular}{|c|c|c|c|c|c|c|c|c|c|}
\hline Parameter & & $\begin{array}{l}\text { Tannins } \\
\text { Dose }\end{array}$ & $\begin{array}{c}\text { Supplementation } \\
\text { Period }\end{array}$ & $\begin{array}{l}\text { Lamb's } \\
\text { Age }\end{array}$ & $\begin{array}{l}\text { Tannins } \\
\text { Type }\end{array}$ & $\begin{array}{l}\text { Tannins } \\
\text { Source }\end{array}$ & $\begin{array}{l}\text { Method of } \\
\text { Inclusion }\end{array}$ & EED & NDFD \\
\hline \multirow[t]{4}{*}{ DWG } & QM & 6.943 & 8.263 & 0.378 & 1.070 & 43.329 & 5.786 & 1.092 & 0.240 \\
\hline & $\mathrm{df}$ & 1 & 1 & 1 & 2 & 32 & 1 & 1 & 1 \\
\hline & $p$-Value & 0.008 & 0.004 & 0.989 & 0.586 & 0.087 & 0.016 & 0.296 & 0.624 \\
\hline & $\mathrm{R}^{2}(\%)$ & 0.54 & 2.85 & 0 & 0 & 0 & 1.17 & 0.92 & 0 \\
\hline \multirow[t]{4}{*}{ DMI } & QM & 4.800 & 10.206 & 0.927 & 2.503 & 113.649 & 0.892 & 0.033 & 2.752 \\
\hline & $\mathrm{df}$ & 1 & 1 & 1 & 2 & 32 & 1 & 1 & 1 \\
\hline & $p$-Value & 0.028 & 0.001 & 0.336 & 0.286 & $<0.001$ & 0.345 & 0.856 & 0.097 \\
\hline & $\mathrm{R}^{2}(\%)$ & 3.02 & 2.49 & 0 & 0 & 14.37 & 0 & 0 & 0 \\
\hline \multirow[t]{4}{*}{ FCR } & QM & 7.711 & 3.716 & 5.348 & 9.193 & 48.362 & 0.129 & 0.006 & 0.335 \\
\hline & $\mathrm{df}$ & 1 & 1 & 1 & 2 & 22 & 1 & 1 & 1 \\
\hline & $p$-Value & 0.005 & 0.050 & 0.021 & 0.010 & $<0.001$ & 0.720 & 0.940 & 0.563 \\
\hline & $\mathrm{R}^{2}(\%)$ & 8.84 & 3.35 & 6.57 & 13.11 & 29.30 & 0 & 0 & 0 \\
\hline \multirow[t]{4}{*}{$\mathrm{HCY}$} & QM & 7.401 & 2.618 & 1.168 & 0.017 & 65.118 & 0.226 & 3.348 & 4.094 \\
\hline & $\mathrm{df}$ & 1 & 1 & 1 & 2 & 22 & 1 & 1 & 1 \\
\hline & $p$-Value & 0.007 & 0.106 & 0.280 & 0.992 & $<0.001$ & 0.634 & 0.067 & 0.043 \\
\hline & $\mathrm{R}^{2}(\%)$ & 14.48 & 4.97 & 1.39 & 0 & 68.77 & 0 & 7.78 & 6.27 \\
\hline \multirow[t]{4}{*}{ LMA } & QM & 0.004 & 5.968 & 13.647 & 0.100 & 36.514 & 0.625 & 2.658 & 2.251 \\
\hline & $\mathrm{df}$ & 1 & 1 & 1 & 1 & 6 & 1 & 1 & 1 \\
\hline & $p$-Value & 0.947 & 0.015 & $<0.001$ & 0.751 & $<0.001$ & 0.429 & 0.103 & 0.134 \\
\hline & $\mathrm{R}^{2}(\%)$ & 0 & 26.30 & 66.18 & 0 & 97.92 & 0 & 8.86 & 8.61 \\
\hline \multirow[t]{4}{*}{ Meat $\mathrm{pH}$} & QM & 0.453 & 1.354 & 0.076 & 17.572 & 68.102 & 6.236 & 0.852 & 0.016 \\
\hline & $\mathrm{df}$ & 1 & 1 & 1 & 2 & 18 & 1 & 1 & 1 \\
\hline & $p$-Value & 0.501 & 0.245 & 0.783 & $<0.001$ & $<0.001$ & 0.013 & 0.356 & 0.899 \\
\hline & $\mathrm{R}^{2}(\%)$ & 0 & 2.62 & 0 & 54.54 & 100 & 23.06 & 0 & 0 \\
\hline \multirow[t]{4}{*}{$\mathrm{L}^{*}$} & QM & 0.132 & 1.913 & 0.728 & 9.171 & 38.080 & 2.731 & 3.146 & 0.126 \\
\hline & $\mathrm{df}$ & 1 & 1 & 1 & 2 & 19 & 1 & 1 & 1 \\
\hline & $p$-Value & 0.716 & 0.167 & 0.393 & 0.010 & 0.006 & 0.098 & 0.076 & 0.723 \\
\hline & $\mathrm{R}^{2}(\%)$ & 0 & 0 & 0 & 11.65 & 26.61 & 1.34 & 0 & 0 \\
\hline \multirow[t]{4}{*}{$a^{*}$} & QM & 0.003 & 13.834 & 16.603 & 4.698 & 29.143 & 0.435 & 12.199 & 10.968 \\
\hline & $\mathrm{df}$ & 1 & 1 & 1 & 2 & 19 & 1 & 1 & 1 \\
\hline & $p$-Value & 0.956 & $<0.001$ & $<0.001$ & 0.095 & 0.064 & 0.509 & $<0.001$ & $<0.001$ \\
\hline & $\mathrm{R}^{2}(\%)$ & 0 & 18.51 & 28.53 & 2.86 & 13.73 & 0 & 19.28 & 21.86 \\
\hline \multirow[t]{4}{*}{$b^{*}$} & $\mathrm{QM}$ & 1.982 & 0.257 & 5.999 & 19.021 & 37.939 & 1.091 & 0.590 & 0.014 \\
\hline & $\mathrm{df}$ & 1 & 1 & 1 & 2 & 19 & 1 & 1 & 1 \\
\hline & $p$-Value & 0.159 & 0.612 & 0.014 & $<0.001$ & 0.009 & 0.296 & 0.442 & 0.906 \\
\hline & $\mathrm{R}^{2}(\%)$ & 0 & 0 & 2.80 & 12.91 & 1.12 & 0 & 0 & 0 \\
\hline \multirow[t]{4}{*}{$\mathrm{CL}$} & QM & 4.339 & 0.121 & 0.471 & 4.947 & 52.306 & 2.199 & 0.121 & 0.036 \\
\hline & $\mathrm{df}$ & 1 & 1 & 1 & 2 & 14 & 1 & 1 & 1 \\
\hline & $p$-Value & 0.037 & 0.728 & 0.492 & 0.084 & $<0.001$ & 0.138 & 0.728 & 0.849 \\
\hline & $\mathrm{R}^{2}(\%)$ & 1.26 & 0 & 0 & 8.34 & 16.48 & 3.11 & 0 & 0 \\
\hline \multirow[t]{4}{*}{ IMF } & $\mathrm{QM}$ & 3.967 & 0.419 & 7.676 & 0.866 & 80.997 & 2.191 & 1.462 & 3.435 \\
\hline & $\mathrm{df}$ & 1 & 1 & 1 & 2 & 18 & 1 & 1 & 1 \\
\hline & $p$-Value & 0.047 & 0.517 & 0.006 & 0.649 & $<0.001$ & 0.139 & 0.227 & 0.064 \\
\hline & $\mathrm{R}^{2}(\%)$ & 9.56 & 0.38 & 14.55 & 0 & 54.24 & 0.33 & 1.96 & 2.56 \\
\hline \multirow[t]{4}{*}{ MDAc } & QM & 9.33 & 11.05 & 56.60 & 29.38 & 143.390 & 9.83 & 13.23 & 0.31 \\
\hline & $\mathrm{df}$ & 1 & 1 & 1 & 2 & 12 & 1 & 1 & 1 \\
\hline & $p$-Value & 0.002 & $<0.001$ & $<0.001$ & $<0.001$ & $<0.001$ & 0.002 & $<0.001$ & 0.574 \\
\hline & $\mathrm{R}^{2}(\%)$ & 17.00 & 1.15 & 56.60 & 32.50 & 93.81 & 5.11 & 4.60 & 4.73 \\
\hline
\end{tabular}

QM: coefficient of moderators; QM was considered significant at $p<0.05 ; \mathrm{R}^{2}$ : amount of heterogeneity accounted for; df: degree of freedom; DWG: daily weight gain; DMI: dry matter intake; FCR: feed conversion ratio; HCY: hot carcass yield; LMA: Longissimus dorsi muscle area; $L^{*}$ lightness; $a^{*}$ : redness: $b^{*}$ : yellowness; CL: cooking loss; IMF: intramuscular fat content; MDAc: malondialdehyde content in raw meat; EED: variation in ether extract content of the diets; NDFD: variation in neutral detergent fiber content of the diets.

Table S3 shows that supplementation with TANs reduced FCR in animals older than three months of age (SMD $=-0.519 ; p<0.01)$, but no effect was observed in lambs up to 
three months of age (SMD $=0.099 ; p>0.05)$. In contrast, LMA increased in sheep older than three months of age (SMD $=1.108 ; p<0.001)$; however, supplementation with TANs did not affect LMA when lambs were up to three months of age (SMD $=-0.245 ; p>0.05$ ). On the other hand, $\mathrm{a}^{*}$ of meat increased when TANs were offered to sheep older than three months of age $(\mathrm{SMD}=0.844 ; p<0.001)$; however, supplementation with TANs did not affect $\mathrm{a}^{*}$ of meat when sheep were up to three months of age (SMD $=-0.061 ; p>0.05$ ). In contrast, meat $b^{*}$ decreased when TANs were offered to sheep up to three months of age $(\mathrm{SMD}=-0.246 ; p<0.05)$, but no significant effects were observed in sheep older than three months of age ( $\mathrm{SMD}=0.502 ; p>0.05$ ). Dietary supplementation with TANs decreased IMF content in animals up to three months of age (SMD $=-0.498 ; p<0.01)$; nevertheless, when TANs were offered to sheep older than three months of age IMF content was not affected $(\mathrm{SMD}=0.553 ; p>0.05)$. Dietary supplementation with TANs reduced MDAc in animals older than three months of age (SMD $=-4.489 ; p<0.001)$, but no effect was observed in lambs up to three months of age (SMD $=-0.320 ; p>0.05)$.

Table 44 shows that FCR decreased in response to dietary supplementation of CTs $(\mathrm{SMD}=-0.563 ; p<0.05)$ and HTs $(\mathrm{SMD}=-2.000 ; p<0.001)$, but there was no change in FCR in sheep supplemented with mixtures of CTs and HTs (SMD $=-0.093 ; p>0.05)$. Meat $\mathrm{pH}$ decreased in response to dietary supplementation with HTs (SMD $=-1.556 ; p<0.001$ ). However, there was no significant change in meat $\mathrm{pH}(p>0.05)$ when CTs $(\mathrm{SMD}=-0.111)$ and mixtures of CTs and HTs (SMD $=0.128)$ were used. $\mathrm{L}^{*}$ of meat increased when HTs were used (SMD $=1.373 ; p<0.05)$, but there was no change $(p>0.05)$ when CTs $(\mathrm{SMD}=-0.072)$ and mixtures of CTs and HTs $(\mathrm{SMD}=-0.114)$ were used. Similarly, $\mathrm{b}^{*}$ of meat increased when HTs were used (SMD $=3.312 ; p<0.05$ ) but decreased when CTs were used (SMD $=-0.500 ; p<0.001)$. However, meat $b^{*}$ was not affected in sheep supplemented with mixtures of CTs and HTs (SMD $=0.123 ; p>0.05)$. Dietary supplementation with mixtures of CTs and HTs decreased MDAc (SMD $=-3.666 ; p<0.001)$, but there was no change $(p>0.05)$ of MDAc in sheep supplemented with CTs (SMD $=-0.313)$ and HTs $(\mathrm{SMD}=-0.106)$.

Table S5 shows that DWG increased when ingredients containing TANs were supplied naturally in the diets (SMD $=0.422 ; p<0.002)$ but DWG was not affected when TANs extracts were used (SMD $=-0.233 ; p>0.05)$. Meat $\mathrm{pH}$ was not affected by the method of inclusion of TANs in the diet $(p>0.05)$. MDAc decreased when ingredients containing TANs were supplied naturally in the diets (SMD $=-2.664 ; p<0.001)$ but was not affected when TANs extracts were used (SMD $=-0.159 ; p>0.05)$.

Figure S2 shows that DMI increased only when TANs came from Mimosa tenuiflora $(\mathrm{SMD}=4.531 ; p=0.001)$, Hedysarum coronarium $(\mathrm{SMD}=7.809 ; p<0.001)$, Ficus infectoria (SMD $=1.448 ; p<0.001)$, Schinopsis spp. ( $\mathrm{SMD}=0.862 ; p=0.026)$, Pistacia vera $(\mathrm{SMD}=0.638 ; p=0.038)$, Passiflora edulis $(\mathrm{SMD}=0.872 ; p=0.044)$ and Prunus amygdalus $(\mathrm{SMD}=0.860 ; p=0.036)$. However, it decreased when TANs came from Cistus ladanifer $(\mathrm{SMD}=-0.363 ; p=0.050)$ and was not affected when TANs came from other plants $(p>0.05)$. Figure 1 shows that FCR decreased when TANs came from plant mixtures $(\mathrm{SMD}=-1.766 ; p=0.017)$, Hedysarum coronarium $(\mathrm{SMD}=-1.140 ; p=0.003)$, Castanea sativa $(\mathrm{SMD}=-2.000 ; p<0.001)$; Vitis vinifera $(\mathrm{SMD}=-2.581 ; p=0.008)$ and Sorghum bicolor $(\mathrm{SMD}=-1.071 ; p=0.008)$. FCR was not affected in sheep supplemented with other sources of TANs $(p>0.05)$.

Figure 2 shows that HCY only increased when TANs were from Hedysarum coronarium $(\mathrm{SMD}=1.675 ; p<0.001)$, Vitis vinifera $(\mathrm{SMD}=0.830 ; p=0.008)$ and Sorghum bicolor $(\mathrm{SMD}=1.440 ; p<0.001)$. However, HCY decreased when TANs were from Mimosa tenuiflora (SMD $=-1.044 ; p=0.037)$. HCY was not affected when other plants were used as sources of TANs $(p>0.05)$. 
Studies

Awawdeh et al. 2019-1

Awawdeh et al. 2019-2

Hassan et al. 2020-3

Pathak et al. 2017-1

Pathak et al. 2017-2

Pathak et al. 2017-3

Subgroup Blend $\left(I^{\wedge} 2=87.28 \%, P=0.000\right)$

Bandeira et al. 2017-3

Bhatt et al. 2020-1

Subgroup Hedysarum coronarium $(1 \wedge 2=0 \%, P=0.547)$

Bhatt et al. 2020-2

Bonanno et al. 2011-

Bonanno et al. 2011-2

Subgroup Ficus infectoria $\left(1^{\wedge} 2=16.53 \%, P=0.302\right)$

Francisco et al. 2018-1

Francisco et al. 2018-2

Guerreiro et al. 2020-1

Guerreiro et al. 2020-2

Guerreiro et al. $2020-3$

Guerreiro et al. 2020-4

Subgroup Cistus ladanifer ( $\left({ }^{\wedge} 2=50.62 \%, P=0.072\right)$

Kamel et al. 2018-1

Kamel et al. 2018-2

Subgroup Schinopsis spp. $\left(\left.\right|^{\wedge} 2=57.55 \%, P=0.125\right)$

Liu et al. 2016-1

Liu et al. 2016-2

Subgroup Castanea sativa $\left(\left(^{\wedge} 2=0 \%, P=0.672\right)\right.$

Flores et al. 2020-1

Flores et al. 2020-2

Flores et al. 2020-3

Subgroup Vitis vinifera $\left(\left.\right|^{\wedge} 2=76.6 \%, P=0.014\right)$

Natalello et al. 2020

Hassan et al. 2020-1

Hatami et al. 2018-

Hatami et al. 2018-2

Subgroup Punica granatum (|^ $2=77.49 \%, \mathrm{P}=0.004)$

Nobre et al. 2020-1

Nobre et al. 2020-2

Nobre et al. 2020-3

Nobre et al. 2020-4

Subgroup Psidium guajava ( $\left.\left.\right|^{\wedge} 2=0 \%, P=0.994\right)$

Norouzian y Ghiasi 2012-1

Norouzian y Ghiasi 2012-2

Norouzian y Ghiasi 2012-3

SoltaniNezhad et al. 2016-1

SoltaniNezhad et al. 2016-2

SoltaniNezhad et al. 2016-3

Subgroup Pistacia vera $\left(\left.\right|^{\wedge} 2=0 \%, P=0.864\right)$

Obeidat et al. 2011-1

Obeidat et al. 2011-2

Subgroup Ceratonia siliqua ( $\left(^{\wedge} 2=0 \%, P=0.881\right)$

Sun et al. 2018-1

un et al. 2018-2

Sun et al. 2018-3

Zhong et al. 2016-1

Zhong et al. 2016-2

Zhong et al 2016-3

Subgroup Sorghum bicolor $\left(\right.$ ( $\left.^{\wedge} 2=58.29 \%, \mathrm{P}=0.035\right)$

Kazemi y Mokhtarpour 2021-1

Kazemi y Mokhtarpour 2021-2

Kazemi y Mokhtarpour 2021-3

Subgroup Prunus amygdalus $\left(\mathrm{I}^{\wedge} 2=0 \%, \mathrm{P}=0.856\right.$

Overall $\left(I^{\wedge} 2=70.15 \%, P=0.000\right)$
Estimate (95\% C.I.)

$0.265 \quad(-0.663,1.192)$

$0.000 \quad(-0.924,0.924)$

$-0.454(-1.342,0.434)$

$-1.576(-2.872,-0.281)$

$-9.189(-13.036,-5.342)$

$-4.498 \quad(-6.624,-2.372)$

$-1.766(-3.222,-0.310)$

$-1.383 \quad(-2.474,-0.292)$

$-0.922(-1.953,0.109)$

$0.148 \quad(-0.985, \quad 1.281)$

$-0.546 \quad(-1.698,0.607)$

$0.750 \quad(-0.421,1.921)$

$0.112 \quad(-0.616$,

$-0.711 \quad(-1.722,0.299)$

$0.166(-0.967,1.300)$

$1.644(0.335,2.953)$

$-0.284 \quad(-1.421, \quad 0.853)$

$0.734 \quad(-0.435,1.903)$

$0.331(-0.320,0.982)$

$0.029(-0.951,-1.010)$

$1.160 \quad(0.100,2.219)$

$0.576(-0.531,1.683)$

$-1.845 \quad(-2.891,-0.798)$

$-2.173 \quad(-3.278,-1.068)$

$-2.000 \quad(-2.760,-1.240)$

$-5.273(-7.667,-2.879)$

$-1.255 \quad(-2.493,-0.017)$

$-2.009 \quad(-3.397,-0.621)$

$-2.581 \quad(-4.486,-0.676)$

$0.739(-0.245, \quad 1.724)$

$\begin{array}{lll}-0.833 \quad(-1.746, & 0.081)\end{array}$

$0.135 \quad(-0.998, \quad 1.268)$

$2.167 \quad(0.741,3.592)$

$0.474 \quad(-0.677$

$-0.283 \quad(-1.268,0.702)$

$-0.427 \quad(-1.418, \quad 0.564)$

$-0.293 \quad(-1.278,0.692)$

$-0.231 \quad(-1.215,0.752)$

$-0.308 \quad(-0.801,0.185)$

$-0.334(-1.389,0.721)$

$0.544 \quad(-0.523,1.610)$

$0.013 \quad(-0.864,0.890)$

$\begin{array}{rrr}-0.238 & (-1.118, & 0.642) \\ 0.036 & (-0.354, & 0.426)\end{array}$

$-0.181 \quad(-1.019,0.656)$

$-0.091 \quad(-0.927,0.745)$

$\begin{array}{lll}-0.136 \quad(-0.728, & 0.456)\end{array}$

$-1.607 \quad(-2.909,-0.306)$

$-1.647 \quad(-2.957,-0.338)$

$-2.560 \quad(-4.086,-1.034)$

$0.391 \quad(-0.751,1.534)$

$-0.828 \quad(-2.007,0.351)$

$-0.615 \quad(-1.773,0.543)$

$-1.071 \quad(-1.867,-0.275)$

$-0.653 \quad(-1.659,0.352)$

$-0.267 \quad(-1.252, \quad 0.717)$

$-0.549(-1.548,0.449)$

$-0.487(-1.062,0.088)$

$-0.444(-0.731,-0.157)$
$0.711 \quad(-0.299,1.722)$

$0.249 \quad(-0.803,1.301)$

$0.095 \quad(-0.782, \quad 0.972)$

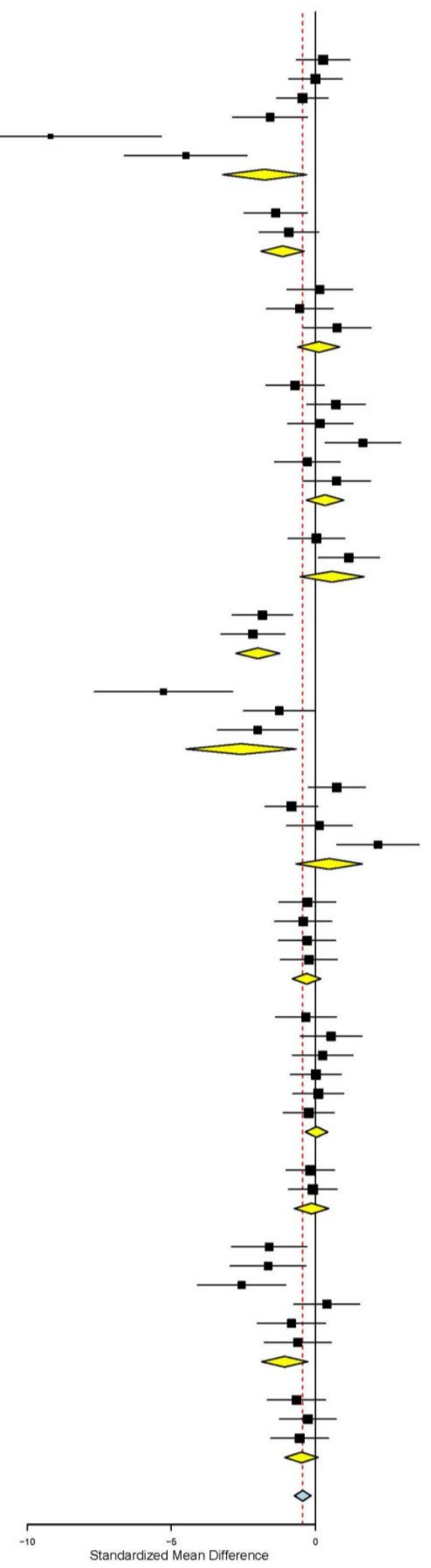

Figure 1. Forest plot of the effect size or standardized mean difference and $95 \%$ confidence interval of the botanical source of tannin on feed conversion ratio (FCR) in sheep. The solid vertical black line represents the mean difference of zero or no effect. Points to the left of the solid vertical black line represent reduction in FCR, while points to the right of the line indicate increase in FCR. 


\section{Studies} Bandeira et al. 2017-2

Bandeira et al. 2017-3

Subgroup Mimosa tenuiflora $\left(\left.\right|^{\wedge} 2=54.56 \%, P=0.111\right)$

Bonanno et al. 2011-1

Bonanno et al. 2011-2

Subgroup Hedysarum coronarium $\left(I^{\wedge} 2=0 \%, P=0.558\right)$

Chikwanha et al 2019b-1

Chikwanha et al. 2019b-2

Chikwanha et al. 2019b-3

Chikwanha et al. 2019b-4

Jerónimo et al. 2010-1

Jerónimo et al. 2010-3

Flores et al. 2020-1

Flores et al. 2020-2

Flores et al. 2020-3

Subgroup Vitis vinifera $\left(I^{\wedge} 2=60.84 \%, P=0.009\right)$

Dentinho et al. 2020

Francisco et al. 2018-1

Francisco et al. 2018-2

Guerreiro et al. 2020-1

Guerreiro et al. 2020-2

Guerreiro et al. 2020-3

Guerreiro et al. $2020-4$

Jerónimo et al. $2010-2$

Jerónimo et al. 2010-4

Subgroup Cistus ladanifer ( $\left(\left.\right|^{\wedge} 2=32.76 \%, P=0.156\right)$

Liu et al. 2016-1

Liu et al. 2016-2

Subgroup Castanea sativa $\left(\left.\right|^{\wedge} 2=0 \%, P=0.701\right)$

Maghaddam et al. 2019-1

Maghaddam et al. 2019-2

Subgroup Berberis vulgaris $\left(\left.\right|^{\wedge} 2=63.51 \%, P=0.098\right)$

Nobre et al. 2020-1

Nobre et al. 2020-2

Nobre et al. 2020-3

Nobre et al. 2020-4

Subgroup Psidium guajava $\wedge^{\wedge} 2=0 \%, P=0.936$ )

Rojas-Román et al. 2017-1

Rojas-Román et al. 2017-2

Rojas-Román et al. 2017-3

Subgroup Blend $\left(I^{\wedge} 2=0 \%, P=0.394\right)$

Sena et al. 2015-1

Sena et al. 2015-2

Sena et al. 2015-3

Subgroup Passiflora edulis $\left(\left.\right|^{\wedge} 2=53.96 \%, P=0.114\right)$

Sharifi y Chaji 2019-1

Sharifi y Chaji 2019-2

Sharifi y Chaji 2019-3

Subgroup Punica granatum $\left(\left.\right|^{\wedge} 2=0 \%, P=0.981\right)$

SoltaniNezhad et al. 2016-1

SoltaniNezhad et al. 2016-2

SoltaniNezhad et al. 2016-3

Subgroup Pistacia vera $\left(I^{\wedge} 2=0 \%, P=0.955\right)$

Zhong et al. 2016-1

Zhong et al. 2016-2

Zhong et al. 2016-3

Subgroup Sorghum bicolor $\left(I^{\wedge} 2=22.38 \%, P=0.276\right)$

Overall $\left(I^{\wedge} 2=52.71 \%, P=0.000\right)$
Bandeira et al. 2017-

Estimate $(958$ C.I.

$-0.361(-1.418, \quad 0.695$

$-0.826(-1.918,0.265)$

$-2.143(-3.457,-0.829)$

$-1.044(-2.028,-0.061)$

$.451(0.350,2.552)$

$1.935(0.747,3.122)$

$1.675(0.867,2.482)$

$-0.063(-1.043,0.917)$

$1.135(0.079,2.191)$

$1.009(-0.031, \quad 2.049)$

$1.387(0.296,2.479)$

$-0.314(-1.453,0.825)$

$-0.488(-1.637,0.660)$

$1.500 \quad(0.219, \quad 2.780$

$1.200(-0.030,2.429)$

$2.727(1.155,4.299)$

$0.830 \quad(0.216, \quad 1.444)$

$0.055(-0.925, \quad 1.035$

$-1.058(-2.105,-0.012)$

$-0.668(-1.675,0.339)$

$-0.406(-1.550,0.737)$

$0.148(-0.985,1.281)$

$0.111(-1.022,1.243)$

$0.148(-0.985, \quad 1.281$

$0.628(-0.531,1.787)$

$1.361(0.105,2.616)$

$-0.011(-0.460,0.438)$

$0.349(-0.534,1.232)$

$0.105(-0.772,0.982)$

$0.226(-0.396,0.849)$

$0.148(-0.901, \quad 1.197)$

$1.483(0.300,2.666)$

$0.786(-0.521,2.094)$

$0.135(-0.846,1.116)$

$0.130(-0.851,1.111)$

$-0.044(-1.024,0.936)$

$0.411(-0.580,1.401)$

$0.157(-0.335,0.648)$

$0.303(-0.579, \quad 1.184$

$0.622(-0.275,1.520)$

$-0.241(-1.121,0.639)$

$0.223(-0.289,0.734)$

$-0.848(-2.142, \quad 0.446)$

$0.404(-0.848,1.656)$

$1.090(-0.238,2.418$

$0.210 \quad(-0.889,1.309)$

$-0.036(-1.016,0.944)$

$-0.054(-1.034,0.926)$

$0.072(-0.908,1.053)$

$-0.006(-0.572,0.560)$

$0.276 \quad(-0.605, \quad 1.157)$

$0.468(-0.421,1.356)$

$0.394(-0.491,1.279)$

$0.379(-0.132,0.889)$

$0.753(-0.418, \quad 1.924)$

$2.260 \quad(0.812,3.709)$

$1.551 \quad(0.261,2.842)$

$1.440 \quad(0.591,2.288)$

$0.350 \quad(0.121, \quad 0.580)$
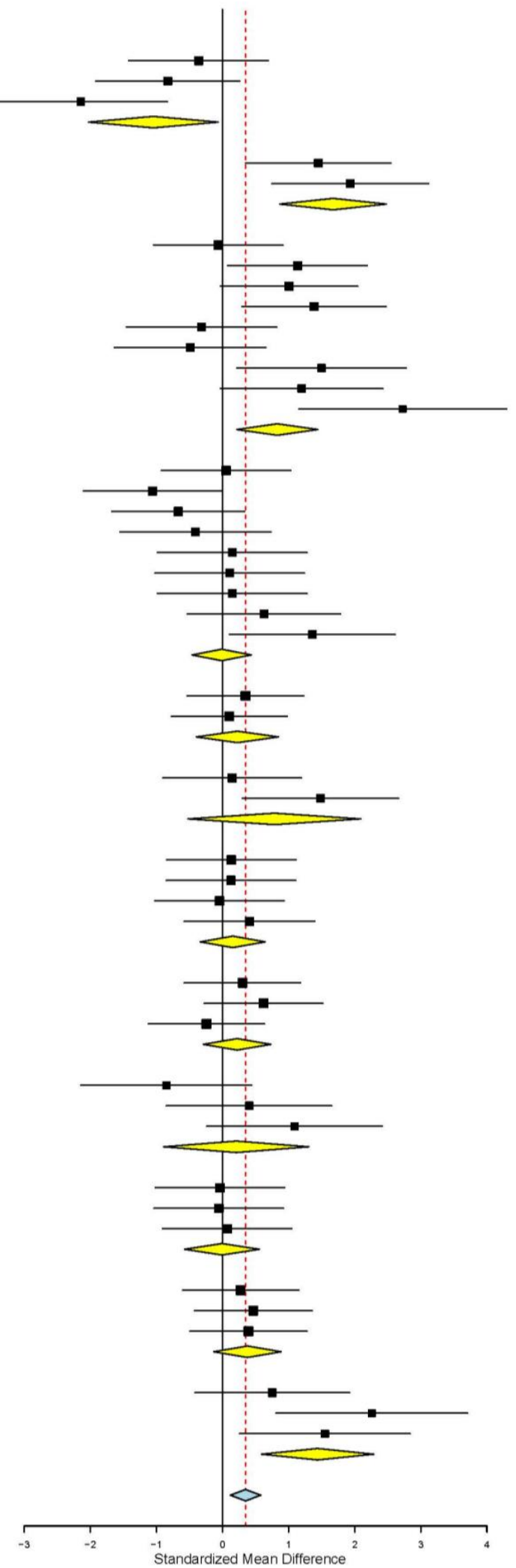

Figure 2. Forest plot of the effect size or standardized mean difference and $95 \%$ confidence interval of the botanical source of tannin on hot carcass yield (HCY) of sheep. The solid vertical black line represents the mean difference of zero or no effect. Points to the left of the solid vertical black line represent reduction in HCY, while points to the right of the line indicate increase in HCY. 
Figure 3 shows that LMA increased only when Pisum sativum (SMD $=0.642 ; p=0.014)$, Vitis vinifera $(\mathrm{SMD}=1.110 ; p=0.021)$ and Pistacia vera $(\mathrm{SMD}=1.774 ; p<0.001)$ were used as sources of TANs. However, LMA was not affected when other plants were used as sources of TANs $(p>0.05)$.

\begin{tabular}{|c|c|c|c|}
\hline Studies & Esti & mate $(958$ & 8 C.I.) \\
\hline Awawdeh et al. $2019-1$ & -0.529 & $(-1.469$ & $0.411)$ \\
\hline Awawdeh et al. 2019-2 & -0.999 & $(-1.979$ & $-0.019)$ \\
\hline Rojas-Román et al. 2017-1 & -0.039 & $(-0.915$ & $0.838)$ \\
\hline Rojas-Román et al. 2017-2 & 0.677 & $(-0.224$ & 1.579) \\
\hline Rojas-Román et al. 2017-3 & 0.094 & $(-0.783$ & $0.971)$ \\
\hline Subgroup Blend $\left(I^{\wedge} 2=43.56 \%, P=0.131\right)$ & -0.138 & $(-0.681$ & $0.406)$ \\
\hline Hart et al. 2011-1 & 1.044 & $(-0.000$ & $2.089)$ \\
\hline Hart et al. 2011-2 & 0.706 & $(-0.374$ & 1. 786$)$ \\
\hline Hart et al. 2011-3 & 0.474 & $(-0.520)$ & $1.468)$ \\
\hline Hart et al. 2011-4 & 0.394 & $(-0.596$ & 1. 383) \\
\hline Subgroup Pisum sativum $\left(1^{\wedge} 2=0 \%, P=0.817\right)$ & 0.642 & $(0.129$ & $1.154)$ \\
\hline Martins et al. $2020-1$ & 1.938 & 10.566 & $3.310)$ \\
\hline Martins et al. $2020-2$ & 0.298 & $(-0.840$ & $1.436)$ \\
\hline Martins et al. $2020-3$ & 1.271 & 10.031 & $2.512)$ \\
\hline Subgroup Vitis vinifera $\left(I^{\wedge} 2=41.29 \%, P=0.182\right)$ & 1.110 & $(0.171$ & $2.048)$ \\
\hline Moghaddam et al. $2019-1$ & 0.005 & $(-1.043$ & 1.053) \\
\hline Moghaddam et al. $2019-2$ & 0.065 & $(-0.982$ & 1.113) \\
\hline Subgroup Berberis vulgaris $\left(1^{\wedge} 2=0 \%, P=0.936\right)$ & 0.035 & $(-0.706$ & $0.776)$ \\
\hline Obeidat e & -0.084 & $(-0.920$ & $0.752)$ \\
\hline Obeidat et al. 2011-2 & -0.378 & $(-1.222$ & $0.465)$ \\
\hline Subgroup Ceratonia siliqua $\left(I^{\wedge} 2=0 \%, P=0.627\right)$ & -0.230 & $(-0.824$ & $0.364)$ \\
\hline SoltaniNezhad et al. 2016-1 & 1.368 & 10.394, & $2.342)$ \\
\hline SoltaniNezhad et al. $2016-2$ & 1.730 & $(0.702$, & $2.757)$ \\
\hline SoltaniNezhad et al. $2016-3$ & 2.394 & (1.246, & 3.542) \\
\hline Subgroup Pistacia vera $\left(I^{\wedge} 2=0 \%, P=0.408\right)$ & 1.774 & $(1.172$, & $2.376)$ \\
\hline Dverall $\left(I^{\wedge} 2=63.42 \%, P\right.$ & 0.497 & $(0.117$ & $0.878)$ \\
\hline
\end{tabular}

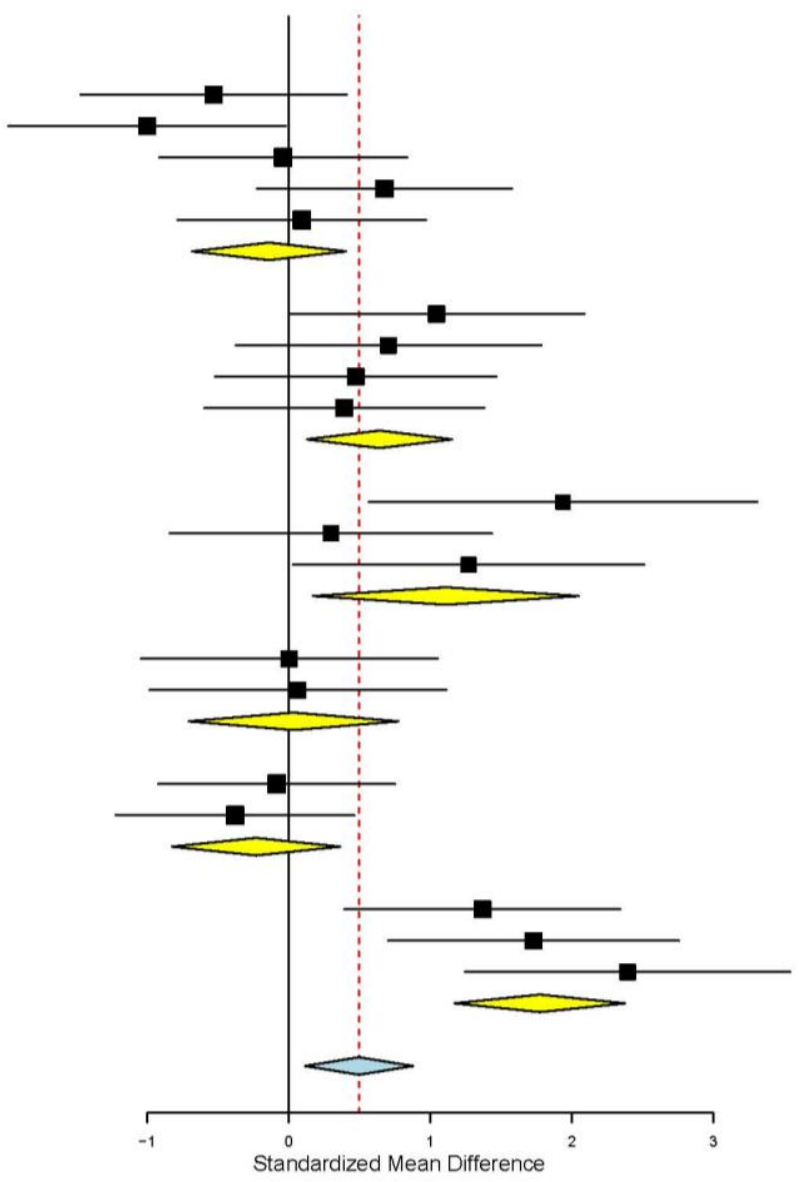

Figure 3. Forest plot of the effect size or standardized mean difference and $95 \%$ confidence interval of the botanical source of tannin on sheep Longissimus dorsi muscle area (LMA). The solid vertical black line represents the mean difference of zero or no effect. Points to the left of the solid vertical black line represent reduction in LMA, while points to the right of the line indicate increase in LMA.

Figure 4 shows that meat $\mathrm{pH}$ increased only when TANs came from Psidium guajava $(\mathrm{SMD}=1.324 ; p<0.001)$, Rosmarinus officinalis $(\mathrm{SMD}=0.866 ; p=0.013)$ and Nigella sativa $(\mathrm{SMD}=0.667 ; p=0.050)$. Meat $\mathrm{pH}$ decreased when TANs were from Castanea sativa $(\mathrm{SMD}=-1.556 ; p<0.001)$, and it was not affected when TANs were from other sources $(p>0.05)$.

Figure S3 shows that $\mathrm{L}^{*}$ of meat increased only when Cistus ladanifer ( $\mathrm{SMD}=0.470$; $p=0.028)$, Castanea sativa (SMD $=1.803 ; p=0.036)$ and Mimosa tenuiflora $(\mathrm{SMD}=0.851$; $p=0.009$ ) were used as sources of TANs. $L^{*}$ decreased when TANs were from Sorghum bicolor $(\mathrm{SMD}=-0.947 ; p=0.007)$, and it was not affected by other sources of TANs $(p>0.05)$. In contrast, Figure $\mathrm{S} 4$ shows that $\mathrm{b}^{*}$ of meat decreased only when Acacia mearnsii (SMD $=-0.884$; $p=0.042)$, Ceratonia siliqua (SMD $=-0.618 ; p=0.045)$ and Sorghum bicolor $(\mathrm{SMD}=-1.358$; $p<0.001$ ) were used as sources of TANs. However, $b^{*}$ increased when TANs were from Mimosa tenuiflora $(\mathrm{SMD}=1.903 ; p=0.033)$, and it was not affected when TANs were from other sources $(p>0.05)$. 


\section{Studies}

Awandeh et al. 2019-1

Awandeh et al. 2019-2

Odhaib et al. 2018-3

Odhaib et al. 2018-6

Odhaib et al. 2018-9

Subgroup Blend $\left(1^{\wedge} 2=0 \%, P=0.820\right)$

Bonanno et al. 2011-1

Bonanno et al. 2011-2

Subgroup Hedysarum coronarium $\left(\left.\right|^{\wedge} 2=0 \%, P=0.813\right)$

Chikwanha et al. 2019b-1

Chikwanha et al. 2019b-2

Chikwanha et al. 2019b-3

Chikwanha et al. 2019-4

Flores et al. 2020-1

Flores et al. 2020-2

Flores et al. 2020-3

Flores et al. 2021-1

Flores et al. 2021-2

Flores et al. 2021-3

Subgroup Vitis vinifera $\left(I^{\wedge} 2=26.84 \%, P=0.197\right)$

Dentinho et al. 2020

Francisco et al. 2018-1

Francisco et al. 2018-2

Guerrero et al. 2020-1

Guerrero et al. 2020-2

Guerrero et al. 2020-3

Guerrero et al. 2020-4

Subgroup Cistus ladanifer $\left(I^{\wedge} 2=0 \%, P=0.831\right)$

Liu et al. 2016-1

Liu et al. 2016-2

Subgroup Castanea sativa $\left(I^{\wedge} 2=7.45 \%, P=0.299\right)$

Nobre et al. 2020-1

Nobre et al. 2020-2

Nobre et al. 2020-3

Nobre et al. 2020-4

Subgroup Psidium guajava $\left(I^{\wedge} 2=0 \%, P=0.801\right)$

Odhaib et al. 2018-1

Odhaib et al. 2018-4

Odhaib et al. 2018-7

Subgroup Rosmarinus officinalis $\left(I^{\wedge} 2=0 \%, P=0.679\right)$

Odhaib et al. 2018-2

Odhaib et al. 2018-5

Odhaib et al. 2018-8

Subgroup Nigella sativa $\left(I^{\wedge} 2=0 \%, P=0.820\right)$

Zhong et al. 2016-1

Zhong et al. 2016-2

Zhong et al. 2016-3

Subgroup Sorghum bicolor $\left(I^{\wedge} 2=0 \%, P=0.614\right)$

Fernandes et al. 2021-1

Fernandes et al. 2021-2

Fernandes et al. 2021-3

Subgroup Mimosa tenuiflora ( $\left({ }^{\wedge} 2=0 \%, P=0.889\right)$

Overall $\left(\left.\right|^{\wedge} 2=47.62 \%, P=0.000\right)$
Estimate (95\% C.I.)

$0.000(-0.924,0.924)$

$0.000(-0.924,0.924)$

$0.000(-1.132, \quad 1.132)$

$0.632(-0.527,1.792)$

$-0.379(-1.521,0.763)$

$0.039(-0.426,0.503)$

$0.167(-0.815, \quad 1.149)$

$0.000(-0.980,0.980)$

$0.083(-0.610,0.777)$

$-0.418(-1.409,0.572)$

$0.084 \quad(-0.897,1.064)$

$0.000(-0.980,0.980)$

$-1.004(-2.044,0.036)$

$-2.275(-3.728,-0.823)$

$-0.758(-1.930,0.413)$

$0.379(-0.763,1.521)$

$0.000(-1.132,1.132)$

$0.000(-1.132,1.132)$

$-0.384(-1.526,0.758)$

$-0.366(-0.773,0.042)$

$0.000(-0.980,0.980)$

$0.338(-0.649,1.325)$

$-0.338(-1.325, \quad 0.649)$

$-0.034(-1.166,1.097)$

$0.011(-1.120,1.143)$

$-0.893(-2.080,0.294)$

$0.011(-1.120,1.143)$

$-0.105(-0.508,0.299)$

$-1.216(-2.170,-0.262)$

$-1.976(-3.045,-0.907)$

$-1.556(-2.297,-0.815)$

$1.668(0.530,2.806)$

$0.927(-0.104,1.958)$

$1.297(0.219,2.376)$

$1.483 \quad(0.376,2.589)$

$1.324(0.781,1.867)$

$0.885(-0.301, \quad 2.070)$

$1.264(0.025,2.503)$

$0.506(-0.644,1.655)$

$0.866(0.179,1.553)$

$0.885(-0.301,2.070)$

$0.758(-0.413,1.930)$

$0.379(-0.763,1.521)$

$0.667(-0.006,1.340)$

$0.000 \quad(-1.132,1.132)$

$-0.589(-1.745, \quad 0.567)$

$-0.785(-1.960,0.389)$

$-0.448(-1.114,0.218)$

$-0.265(-1.317,0.787)$

$-0.177(-1.226,0.873)$

$0.088(-0.960,1.136)$

$-0.117(-0.724,0.489)$

$0.009(-0.222, \quad 0.241)$
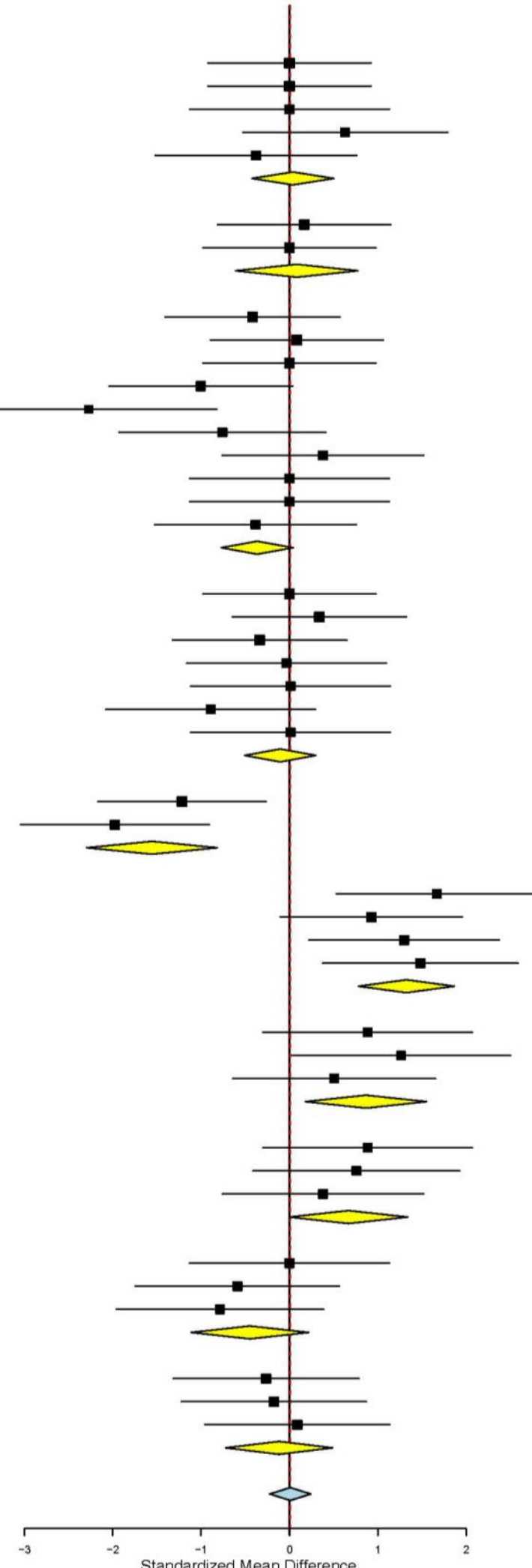

Figure 4. Forest plot of the effect size or standardized mean difference and $95 \%$ confidence interval of the botanical source of tannin on meat $\mathrm{pH}$ of sheep. The solid vertical black line represents the mean difference of zero or no effect. Points to the left of the solid vertical black line represent reduction in meat $\mathrm{pH}$, while points to the right of the line indicate increase in meat $\mathrm{pH}$. 
Figure 5 shows that the IMF content of meat decreased only when Cesalpinia spinosa $(\mathrm{SMD}=-0.870 ; p=0.028)$ and Onobrychis viciifolia $(\mathrm{SMD}=-1.319 ; p=0.042)$ were used as sources of TANs. However, IMF increased when TANs were from Vitis vinifera $(\mathrm{SMD}=0.893 ; p=0.050)$, and it was not affected when TANs were from other plants $(p>0.05)$. Additionally, Figure S5 shows that CL decreased only when TANs came from Vitis vinifera (SMD $=-1.584 ; p=0.047$ ) and Rosmarinus officinalis (SMD $=-0.691 ; p=0.045)$. CL increased when TANs came from Castanea sativa (SMD $=1.949 ; p<0.001$ ) and Psidium guajava (SMD $=6.842 ; p<0.001$ ), and it was not affected when TANs came from other sources $(p>0.05)$.

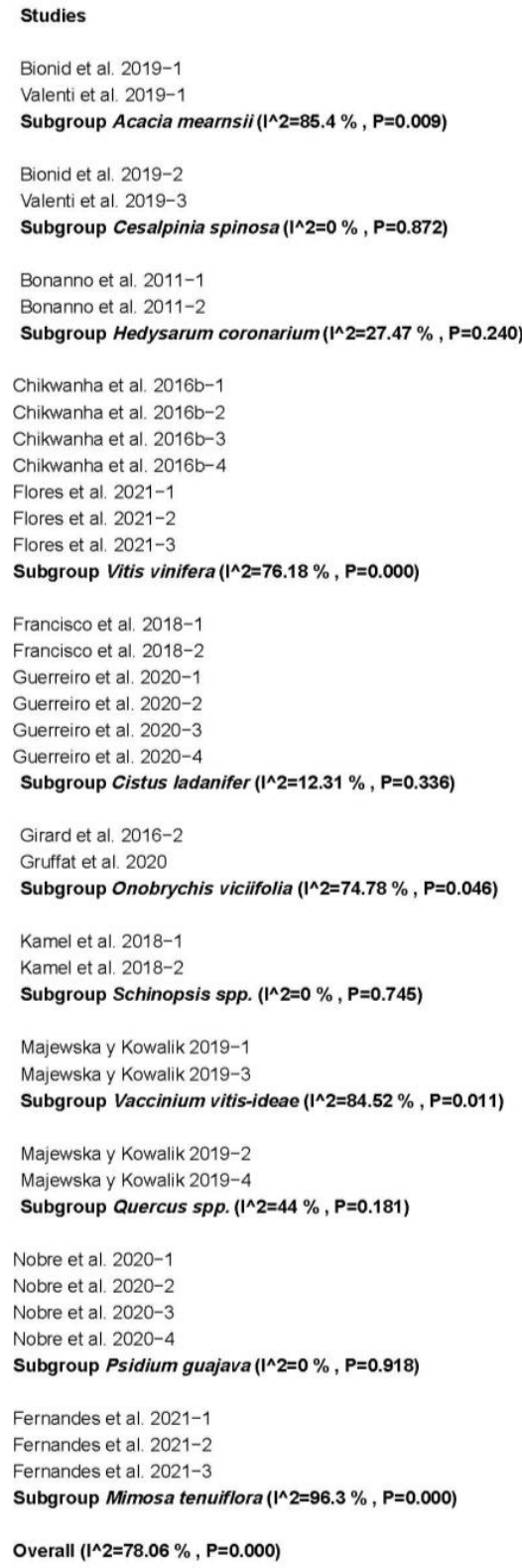

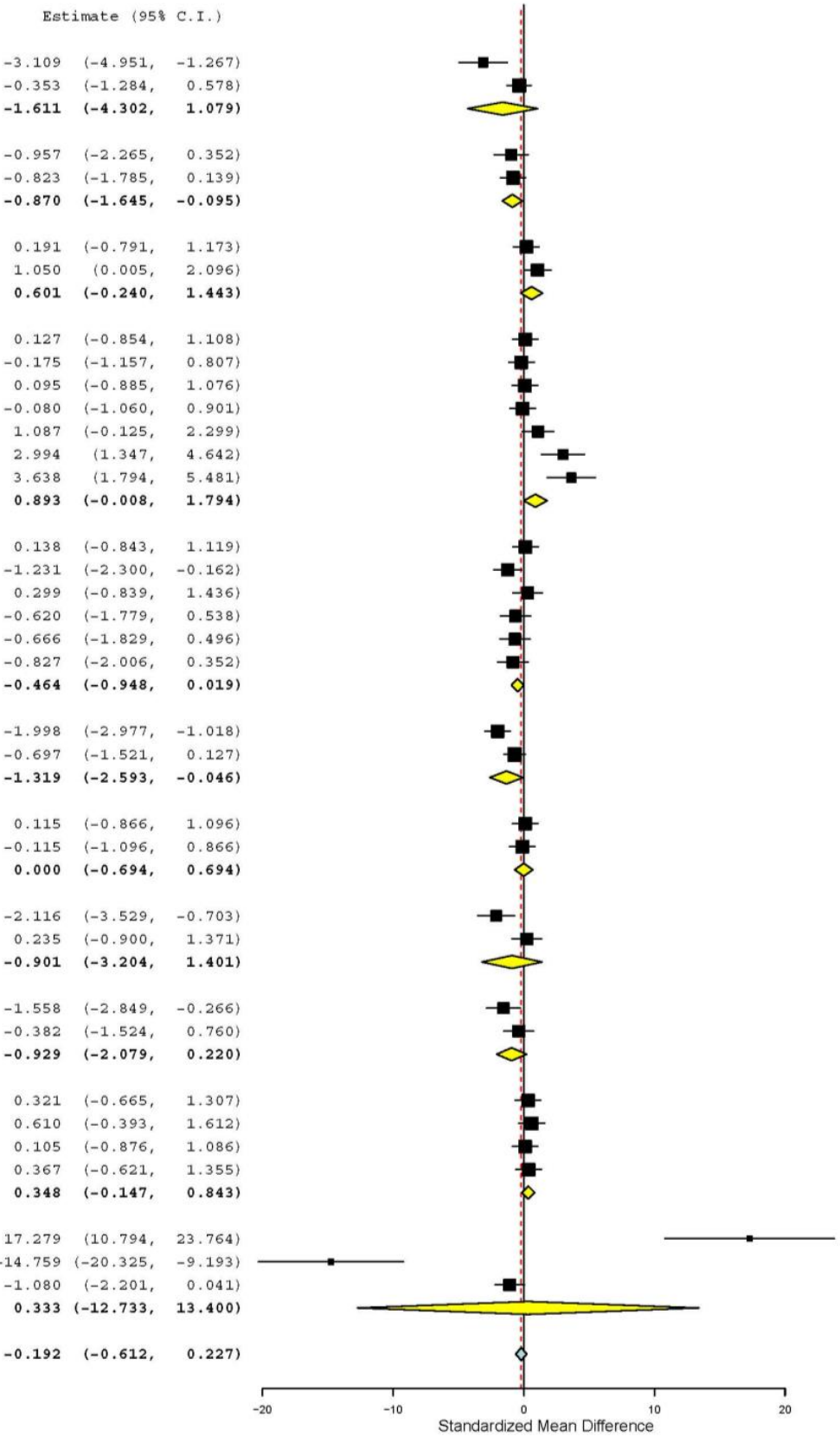

Figure 5. Forest plot of the effect size or standardized mean difference and 95\% confidence interval of the source of botanical origin of tannin on intramuscular fat (IMF) content in sheep meat. The solid vertical black line represents the mean difference of zero or no effect. Points to the left of the solid vertical black line represent reduction in IMF, while points to the right of the line indicate increase in IMF. 
Figure 6 shows that MDAc content decreased only when TANs came from Vitis vinifera $(\mathrm{SMD}=-3.106 ; p<0.001)$, Rosmainus officinalis $(\mathrm{SMD}=-5.479 ; p<0.001)$, Nigella sativa $(\mathrm{SMD}=-6.022 ; p<0.001)$, Sorghum bicolor (SMD $=-0.843 ; p=0.017)$ and plant mixtures $(\mathrm{SMD}=-5.184 ; p<0.001)$. While MDAc was not affected when TANs were from other plants $(p>0.05)$.

\section{Studies}

Biondi et al. 2019-1

Valenti et al. 2019-1

Subgroup Acacia mearnsii $\left(\left.\right|^{\wedge} 2=0 \%, P=0.862\right)$

Biondi et al. 2019-2

Valenti et al. 2019-3

Subgroup Cesalpinia spinosa $\left(I^{\wedge} 2=0 \%, P=0.890\right)$

Francisco et al. 2018-1

Francisco et al. 2018-2

Jeronimo et al. 2012-2

Subgroup Cistus ladanifer ( $\left(\left.\right|^{\wedge} \mathbf{2}=\mathbf{2 2 . 8 1} \%, \mathrm{P}=\mathbf{0 . 2 7 4}\right)$

Gruffat et al. 2020-1

Gruffat et al. 2020-2

Subgroup Onobrychis viciifolia $\left(I^{\wedge} 2=0 \%, P=0.500\right)$

Jerónimo et al. 2012-1

Martins et al. 2021-1

Martins et al. 2021-2

Martins et al. 2021-3

Subgroup Vitis vinifera $\left(\left.\right|^{\wedge} 2=73.34 \%, P=0.010\right)$

Odhaib et al. 2018-1

Odhaib et al. 2018-4

Odhaib et al. 2018-7

Subgroup Rosmarinus officinalis $\left(\left.\right|^{\wedge} 2=19.75 \%, P=0.288\right)$

Odhaib et al. 2018-2

Odhaib et al. 2018-5

Odhaib et al. 2018-8

Subgroup Nigella sativa $\left(\left.\right|^{\wedge} 2=0 \%, P=0.465\right)$

Odhaib et al. 2018-3

Odhaub et al. 2018-6

Odhaib et al. 2018-9

Subgroup Blend $\left(I^{\wedge} 2=0 \%, P=0.783\right)$

Zhong et al. 2016-1

Zhong et al. 2016-2

Zhong et al. 2016-3

Subgroup Sorghum $\left(I^{\wedge} 2=0 \%, P=0.388\right)$

Overall $\left(I^{\wedge} 2=87.22 \%, P=0.000\right)$

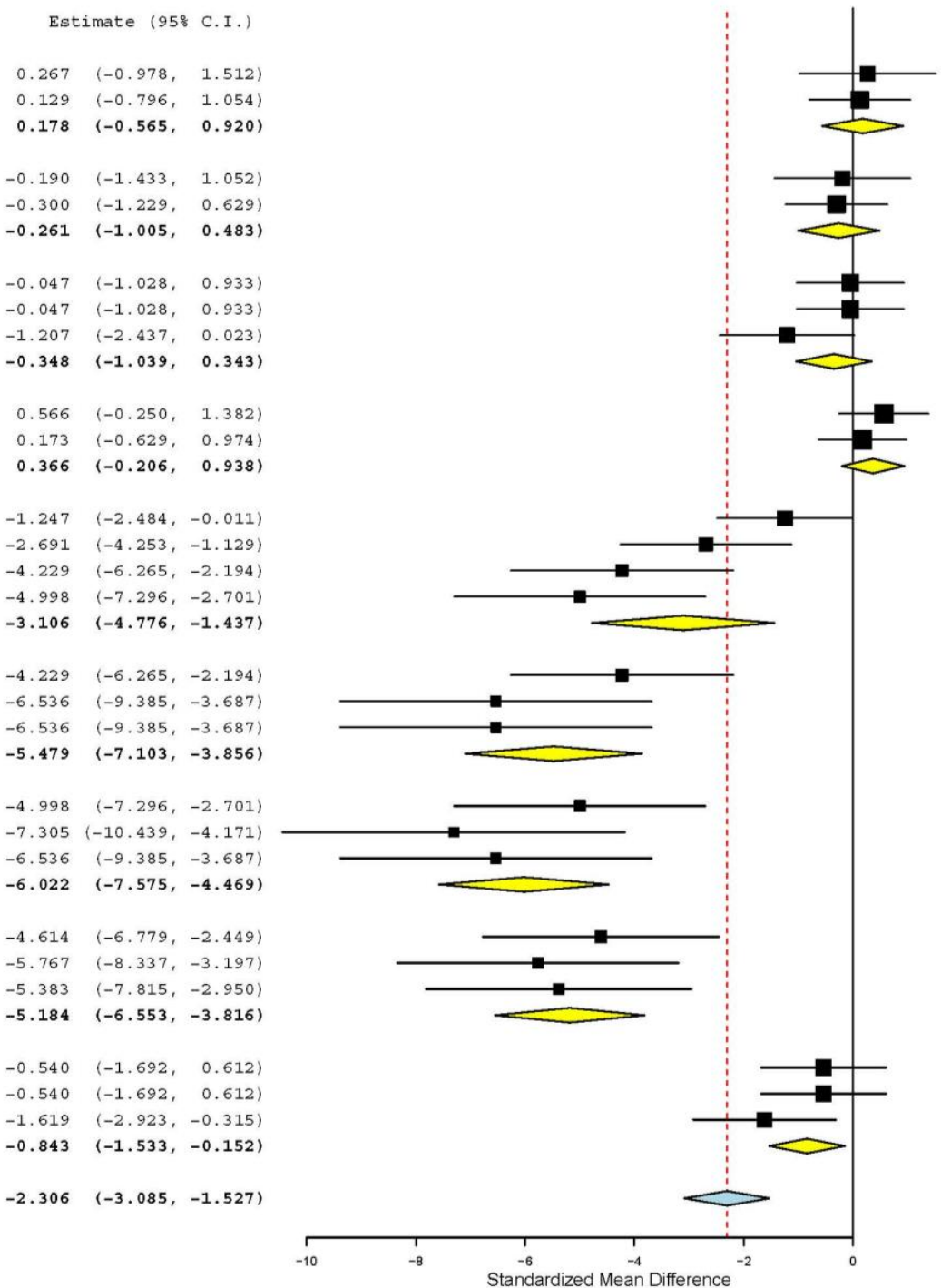

Figure 6. Forest plot of the effect size or standardized mean difference and 95\% confidence interval of the source of botanical origin of tannin on the malondialdehyde content of raw sheep meat (MDAc). The solid vertical black line represents the mean difference of zero or no effect. Points to the left of the solid vertical black line represent reduction of MDAc, while points to the right of the line indicate increase of MDAc.

\section{Discussion}

It has been suggested that high doses of TANs in the diet $\left(>55 \mathrm{~g} \mathrm{~kg}^{-1} \mathrm{DM}\right)$ may have negative effects on feed utilization efficiency and growth rate of ruminants; however, lowmoderate levels ( 20 to $40 \mathrm{~g} \mathrm{~kg}^{-1} \mathrm{DM}$ ) could result in neutral or even positive effects [9,12]. In this regard, a meta-analysis conducted by Orzuna-Orzuna et al. [32] reported that dietary supplementation with TANs at average doses of $14.61 \mathrm{~g} \mathrm{~kg}^{-1} \mathrm{DM}$ did not affect DWG or feed efficiency in beef cattle. However, in the present meta-analysis, higher DWG and lower FCR were observed in response to dietary supplementation with TANs. This suggests that TANs improve growth rate and feed utilization efficiency in sheep. The lower FCR observed could be explained because TANs supplementation reduces infection by 
gastrointestinal nematodes, which decrease feed efficiency in livestock [1]. According to Pimentel et al. [12], dietary inclusion of TANs in moderate doses can increase the efficiency of nutrient utilization of the diet, due to the ability of TANs to form complexes with macromolecules. In addition, the presence of TANs in diets consumed by ruminants has been reported to reduce enteric methane production [32], increase duodenal flux of amino acids and microbial protein [51], and reduce energy losses due to urea excretion [32,52]. This would partially explain the positive effects observed in DWG and FCR for sheep supplemented with TANs. On the other hand, reactive oxygen species (ROS) oxidize and destroy cellular biological molecules and impair the integrity of the intestinal membrane, resulting in reduced nutrient absorption [53,54]. Antioxidant enzymes and exogenous antioxidants can help restore oxidative balance and maintain healthy intestinal mucosa $[21,54,55]$. Consequently, the positive effects observed for DWG and FCR in the present study suggest that TANs reduced the presence of ROS and intestinal membrane damage in sheep. This hypothesis is supported by the observed increase in CAT and GPx in sheep supplemented with TANs, because CAT and GPx can convert ROS into less harmful compounds for the organism and prevent lesions in the gastrointestinal mucosa $[53,56]$.

Although some review articles have suggested that the presence of TANs in the diet may negatively affect DMI in ruminants $[1,9,57]$, in the present meta-analysis no changes in DMI were observed in response to dietary supplementation with TANs. This absence of changes in DMI probably occurred because the average dose of TANs used was $19.29 \mathrm{~g} \mathrm{~kg}^{-1} \mathrm{DM}$ and negative effects of TANs on intake seem to occur with doses higher than $50 \mathrm{~g} \mathrm{~kg}^{-1} \mathrm{DM}[4,9,12]$. Similar to our results, two previously elaborated metaanalyses reported that dietary supplementation with TANs at average concentrations of 9.5 and $14.61 \mathrm{~g} \mathrm{~kg}^{-1} \mathrm{DM}$ did not significantly affect DMI of dairy cows in production and beef cattle, respectively [31,32]. These results together suggest that TANs can be used in sheep and cattle without negative effects on feed intake.

It has been suggested that the reduction of feed palatability could be produced by a reaction between dietary TANs and salivary mucoproteins, or by a direct reaction of TANs with taste receptors, causing an astringent sensation [10,58,59]. However, prolonged exposure to dietary TANs can induce adaptive mechanisms in ruminants, such as changes in the amount of proline and other salivary proteins with high affinity for TANs [8,59-61]. Unlike other protein complexes and TANs formed, protein complexes rich in proline and TANs are stable over the entire $\mathrm{pH}$ range of the digestive tract, which could eliminate or reduce their negative effect on palatability and feed intake [10,62-64]. Regarding this, in the present study a subgroup analysis revealed that DMI was not affected when TANs were offered for up to 70 days but increased when supplementation lasted more than 70 days.

With respect to carcass characteristics, dietary supplementation with TANs increased HCY and CCY. There is limited information on the effects of TANs on ruminant carcass characteristics, which makes it difficult to explain the results observed in the present meta-analysis. However, variation in carcass fat and muscle deposition can modify carcass performance [65]. Consequently, the observed increase in LMA and BFT in the carcasses of sheep supplemented with TANs could partially explain the higher $\mathrm{HCY}$ and $\mathrm{CCY}$ obtained in the present meta-analysis.

BFT and LMA also increased in response to dietary supplementation with TANs. The mechanism of action of TANs on lipogenesis and muscle development has not been studied in sheep. Nevertheless, some polyphenolic compounds have been reported to increase BFT in beef cattle by changing the differential expression of genes involved in lipid metabolism [66]. Similarly, dietary supplementation from plants with phenolic compounds can increase muscle fiber size and increase skeletal muscle mass in lambs [67]. Similar effects of TANs consumption in the present meta-analysis would partially explain the observed increases in BFT and LMA.

Meat with high $\mathrm{pH}$ has higher microbial deterioration, which reduces its quality and shelf life [50]. Although dietary supplementation with TANs did not affect meat $\mathrm{pH}$, subgroup analysis revealed that meat $\mathrm{pH}$ decreased significantly when HTs were used. 
This suggests that HTs could reduce microbial spoilage of meat, and consequently improve its quality and increase its shelf life. In this regard, Biondi et al. [68] observed lower overall load of pathogenic bacteria (Escherichia coli, Enterobacteriaceae and Pseudomonas spp.) in meat from lambs supplemented with HTs $\left(40 \mathrm{~g} \mathrm{~kg}^{-1} \mathrm{DM}\right)$ of Caesalpinia spinosa and concluded that HTs may have antimicrobial activity within muscle tissue.

Color is one of the most important characteristics that determines meat quality, because it is the first attribute that attracts consumers when choosing fresh meat $[50,69]$. The $\mathrm{pH}$, IMF content, the amount of myoglobin, and the formation and accumulation of muscle metmyoglobin are the main factors that influence the color of small ruminant meat [65]. Particularly, $\mathrm{L}^{*}$ of meat depends on the IMF content [70] and is negatively correlated $(\mathrm{r}=-0.63)$ with muscle myoglobin concentration [71]. Similarly, $\mathrm{b}^{*}$ of meat is correlated with $\mathrm{pH}$ [72], and with the IMF content of meat [70]. In the present metaanalysis, $\mathrm{pH}$ and meat IMF content were similar between treatments. In addition, previous studies $[17,18]$ have reported that dietary supplementation of TANs does not affect muscle myoglobin content in sheep. These findings could partially explain the absence of $L^{*}$ and $b^{*}$ changes observed in sheep consuming TANs in the present study. Additionally, compounds originating in meat as a consequence of lipid oxidation have been reported to promote the formation of metmyoglobin, which reduces $a^{*}$ values in meat [73]. In the present meta-analysis, MDAc (as an indicator of lipid oxidation in meat) and the metmyoglobin content of meat decreased in response to dietary supplementation with TANs, which would partially explain the observed increase in $a^{*}$ in meat from sheep supplemented with TANs.

Meat tenderness is one of the main characteristics that influences consumers' meat choice and can be evaluated by WBSF [65]. It has been hypothesized that some natural antioxidants, such as polyphenols extracted from citrus fruits, might contain tenderizing compounds because their use can increase meat tenderness [74]. However, although TANs are polyphenolic compounds with antioxidant activity [75], in the present study the addition of TANs in the sheep diet did not affect WBSF. These results suggest that TANs do not affect the tenderness of sheep meat. Malheiros et al. [76] reported that ROS production can improve meat tenderness by increasing the degradation of toughness-related structural proteins. It has been reported that TANs can improve the antioxidant status of small ruminant meat by increasing mRNA and protein expression levels of SOD and GPx in skeletal muscle cells [77]. This effect could reduce structural and functional damage in muscle cells and tissues caused by ROS [78], which would partially explain the absence of changes observed for WBSF in the present study.

DL and CL are parameters used to evaluate the water holding capacity (WHC) of meat [65]. In the present meta-analysis, the values observed for DL suggest that dietary supplementation with TANs can improve WHC. However, these results should be interpreted carefully due to the low number of studies that reported on this variable. There is a strong negative correlation $(r=-0.894)$ between WHC and CL [79]. Therefore, the results observed for CL suggest that TANs do not affect WHC of sheep meat. Meat $\mathrm{pH}$ is also related to WHC $[50,65]$, and variation in IMF content can alter muscle structure and modify water retention in meat [65]. This suggests that the similarity of IMF content and meat $\mathrm{pH}$ between treatments could be related to the lack of changes observed for CL in the present meta-analysis.

Meat moisture content decreased in response to dietary supplementation with TANs, suggesting that TANs might affect WHC of meat. However, these results should be interpreted carefully due to the low number of studies that reported on this variable. Moreover, the results observed for protein, IMF and ash content of meat indicate that supplementation with TANs does not affect the nutritional composition of sheep meat.

Subgroup analysis revealed that doses of TANs higher than $20 \mathrm{~g} \mathrm{~kg}^{-1} \mathrm{DM}$ can reduce IMF content. The mechanism of action of TANs on IMF deposition has not been studied in sheep. However, the number and size of intramuscular adipocytes have been reported to be related to the process of IMF deposition or reduction in livestock $[80,81]$. Gallic acid (a typical isomer of HTs) can inhibit bovine adipocyte proliferation and adipogenesis under 
in-vitro conditions [81]. Similarly, in-vitro studies have reported that CTs and HTs inhibit preadipocyte differentiation [82,83], and CTs induce apoptosis in mature adipocytes and inhibit lipid accumulation in maturing preadipocytes [82]. This would partly explain the lower IMF content in meat from sheep supplemented with more than $20 \mathrm{~g} \mathrm{~kg}^{-1} \mathrm{DM}$ of TANs. The lower IMF content could be related to the reduced moisture content of meat from sheep supplemented with TANs, because there is a negative correlation $(\mathrm{r}=-0.47)$ between moisture content and IMF content of meat [84].

Oxidation reactions during the processing, distribution and storage of meat products can cause physicochemical changes and undesirable odors that affect the quality of the final product [85]. For example, oxidation of myoglobin and lipids can cause discoloration and off-flavor development in meat, respectively [86]. In the present meta-analysis, lipid oxidation and myoglobin oxidation of meat decreased in response to dietary supplementation with TANs. This suggests that TANs may induce antioxidant enzyme gene expression in sheep muscle [87], similar to what was previously observed by Zhong et al. [77] in skeletal muscle cells from goat treated with Camellia sinensis TANs under in-vitro conditions. These results also suggest that TANs could be used to delay the discoloration and appearance of off-flavor in meat, and consequently improve the quality and shelf life of meat products.

TANs are polyphenolic compounds with antioxidant activity [75]. It has been reported that phenols can switch from an antioxidant to a prooxidant state when used in high doses [88]. In the present meta-analysis, lipid oxidation of meat decreased in response to dietary supplementation with TANs regardless of the dose used. However, the reduction was greater at doses below $20 \mathrm{~g} \mathrm{~kg}^{-1} \mathrm{DM}$. These results suggest that TANs may improve the oxidative stability of meat when supplied to the diet at low doses, but at high concentrations they could have prooxidant effects on meat, as previously reported for some essential oils rich in phenolic compounds [88].

Subgroup analysis revealed that lipid oxidation of meat decreased significantly only when TANs were fed to animals older than 3 months of age. This probably occurred because the presence of microorganisms in the rumen increases with the age of the animals [89], and the action of ruminal microorganisms increases the bioavailability of ingested TANs in sheep [90].

The type and source of TANs explained most (between 30 and 90\%) of the heterogeneity observed in MDAc. These results suggest that the effects of TANs on oxidative stability of meat depend on the type and source of TANs used rather than the dose, period and method of supplementation. Subgroup analyses revealed that MDAc was significantly reduced only when mixtures of CTs and HTs were used. These results suggest a synergistic effect between both types of TANs in reducing lipid oxidation of meat. Although TANs have been shown to have high antioxidant activity [75], their effect on the antioxidant capacity of muscle tissues seems to depend on how effectively they can be absorbed through the gastrointestinal tract [91]. In this regard, it has been reported that some CTs can neither be degraded nor absorbed in the gastrointestinal tract of sheep [91]. In contrast, some HTs act for a short time because they are rapidly transferred to the blood plasma in sheep [90]. Similar effects of individual use of CTs and HTs in sheep would partially explain the results observed in the present meta-analysis.

TAC is an integrated parameter that considers all antioxidants present in blood plasma [92]. In the present study, TAC was observed to increase in response to dietary supplementation with TANs, suggesting that TANs intake may improve the total antioxidant status of sheep. Although there are no reference values for TAC in ruminants [93], TAC changes in blood plasma following supplementation with antioxidant-rich foods or purified antioxidants provide information on the absorption and bioavailability of ingested antioxidant compounds [92]. Therefore, although the metabolic fate of TANs ingested by ruminants is not yet fully understood [22], the results observed in the present meta-analysis suggest that TANs ingested by sheep may be degraded and absorbed in the gastrointestinal tract and subsequently transferred to the bloodstream to serve as exogenous antioxidants. 
Excessive accumulation of prooxidant substances, such as ROS, can cause oxidative stress in ruminants $[93,94]$. Some antioxidant enzymes, such as GPx, SOD and CAT are important because they can convert ROS into less harmful compounds for the organism [56], and consequently can reduce ROS-mediated damage on biological macromolecules $[95,96]$. On the other hand, although ROS can attack any of the major biomolecules, lipids are particularly susceptible, so biomarkers of lipid peroxidation (e.g., malondialdehyde) are considered the best indicators of oxidative stress [97]. In the present meta-analysis, dietary supplementation with TANs increased the activity of antioxidant enzymes (CAT and GPx) and reduced the concentration of malondialdehyde in sheep blood serum. This suggests that inclusion of TANs in the diet could be used as a dietary strategy to mitigate oxidative stress in sheep, which could improve animal health [94].

\section{Conclusions}

The results of the present meta-analysis indicate that dietary supplementation with TANs does not affect dry matter intake, but improves daily weight gain, feed conversion ratio, total antioxidant capacity and antioxidant enzyme activity in sheep blood serum. The best result for daily weight gain is achieved using doses of TANs lower than $20 \mathrm{~g} \mathrm{~kg}^{-1} \mathrm{DM}$, with supplementation periods longer than 70 days and when TANs are supplied naturally as ingredients in the diet. The best results for feed conversion ratio are achieved using doses of TANs lower than $20 \mathrm{~g} \mathrm{~kg}^{-1} \mathrm{DM}$, with supplementation periods longer than 70 days, in animals older than three months of age and using CTs.

In addition, TANs reduce lipid oxidation in blood plasma and meat. The best results of lipid oxidation of meat are observed using mixtures of CTs and HTs, with supplementation periods longer than 70 days, using doses lower than $20 \mathrm{~g} \mathrm{~kg}^{-1} \mathrm{DM}$, in animals older than three months of age, and when TANs are supplied naturally as ingredients in the diet. Supplementation with TANs does not affect meat tenderness, chemical composition, $\mathrm{pH}$ and color $\left(\mathrm{L}^{*}\right.$ and $\left.\mathrm{b}^{*}\right)$. However, it increases hot and cold carcass yield, backfat thickness and Longissimus dorsi muscle area. The best hot carcass yield is obtained with Vitis vinifera, Hedysarium coronarium and Sorghum bicolor as sources of TANs. In contrast, the best Longissimus dorsi muscle area result is observed in animals older than three months of age, with supplementation periods longer than 70 days, and using Vitis vinifera, Pisum sativum and Pistacia vera as sources of TANs. In addition, supplementation with TANs improves meat $\mathrm{a}^{*}$, particularly with supplementation periods longer than 70 days and in animals older than three months of age.

Supplementary Materials: The following are available online at https: / / www.mdpi.com/article/ 10.3390/ani11113184/s1, Figure S1: A PRISMA flow diagram detailing the literature search strategy and study selection for the meta-analysis. Table S1: Subgroup analysis of the effect of dietary tannin dose in sheep. Table S2: Subgroup analysis of the effect of tannin supplementation period in sheep. Table S3: Subgroup analysis of the effect of age on the response to tannin supplementation in sheep. Table S4: Subgroup analysis of the effect of tannin type in sheep. Table S5: Subgroup analysis of the effect of tannin inclusion method in sheep. Figure S2: Forest plot of the effect size or standardized mean difference and $95 \%$ confidence interval of the source of botanical origin of tannin on dry matter intake (DMI) in sheep. Figure S3: Forest plot of the effect size or standardized mean difference and $95 \%$ confidence interval of the botanical source of tannin on the lightness $\left(L^{*}\right)$ of sheep meat. Figure S4: Forest plot of the effect size or standardized mean difference and $95 \%$ confidence interval of the botanical source of tannin on the yellowness $\left(b^{*}\right)$ of sheep meat. Figure S5: Forest plot of the effect size or standardized mean difference and $95 \%$ confidence interval of the botanical source of tannin on cooking loss (CL) of sheep meat.

Author Contributions: Conceptualization, J.F.O.-O., G.D.-I. and A.L.-B.; methodology and data curation, J.F.O.-O., G.D.-I. and G.D.M.-M.; investigation and formal analysis, J.F.O.-O., L.A.M.-R. and H.A.L.-R.; writing-original draft preparation, J.F.O.-O.; writing-review and editing, J.F.O.-O., G.D.-I., A.L.-B., G.D.M.-M., L.A.M.-R. and H.A.L.-R.; supervision and project administration, A.L.-B. All authors have read and agreed to the published version of the manuscript. 
Funding: This research received no external funding.

Institutional Review Board Statement: Not applicable.

Informed Consent Statement: Not applicable.

Data Availability Statement: The datasets used and analyzed during the current study are available from the corresponding author on reasonable request.

Acknowledgments: The first author, José Felipe Orzuna Orzuna is a Master of Science student of the Program of Animal Production of the Universidad Autónoma Chapingo and thanks the CONACyT Program for the scholarship.

Conflicts of Interest: The authors declare no conflict of interest.

\section{Appendix A}

Table A1. Summary of the studies included in the meta-analysis.

\begin{tabular}{|c|c|c|c|c|}
\hline Author & Country & Tannin Source & Tannin Type & Method of Inclusion \\
\hline Awawdeh et al. [98] & Jordan & $\mathrm{B}, \mathrm{B}$ & B, B & $\mathrm{N}, \mathrm{N}$ \\
\hline Bandeira et al. [99] & Brazil & Mimosa tenuiflora $(n=3)$ & $\mathrm{CT}, \mathrm{CT}, \mathrm{CT}$ & $\mathrm{N}, \mathrm{N}, \mathrm{N}$ \\
\hline Bhatt et al. [100] & India & PA, ER & B, B & $\mathrm{N}, \mathrm{N}$ \\
\hline Biondi et al. [68] & Spain & $\mathrm{AM}, \mathrm{CS}$ & B, B & $\mathrm{E}, \mathrm{E}$ \\
\hline Bonanno et al. [101] & Italy & Hedysarum coronarium & $\mathrm{CT}, \mathrm{CT}$ & $\mathrm{N}, \mathrm{N}$ \\
\hline Buccioni et al. [102] & Italy & $\mathrm{CH}, \mathrm{QU}$ & $\mathrm{HT}, \mathrm{CT}$ & $\mathrm{E}, \mathrm{E}$ \\
\hline Chikwanha et al. [103] & South Africa & $\operatorname{VV}(n=4)$ & $\mathrm{B}, \mathrm{B}, \mathrm{B}, \mathrm{B}, \mathrm{B}$ & $\mathrm{N}, \mathrm{N}, \mathrm{N}, \mathrm{N}, \mathrm{N}$ \\
\hline Chikwanha et al. [104] & South Africa & $\operatorname{VV}(n=4)$ & B, B, B, B, B & $\mathrm{N}, \mathrm{N}, \mathrm{N}, \mathrm{N}, \mathrm{N}$ \\
\hline Costa et al. [105] & Brazil & $\mathrm{AM}(n=4)$ & $\mathrm{CT}, \mathrm{CT}, \mathrm{CT}, \mathrm{CT}, \mathrm{CT}$ & $\mathrm{E}, \mathrm{E}, \mathrm{E}, \mathrm{E}, \mathrm{E}$ \\
\hline Dentinho et al. [20] & Brazil & CL & $\mathrm{CT}$ & $\mathrm{E}$ \\
\hline Dey et al. [106] & India & Ficus infectoria $(n=3)$ & $\mathrm{B}, \mathrm{B}, \mathrm{B}$ & $\mathrm{N}, \mathrm{N}, \mathrm{N}$ \\
\hline Abdalla et al. [107] & Brazil & OP, Clep & B, B & $\mathrm{N}, \mathrm{N}$ \\
\hline Fernandes et al. [14] & Brazil & Mimosa tenuiflora $(n=3)$ & $\mathrm{B}, \mathrm{B}, \mathrm{B}$ & $\mathrm{N}, \mathrm{N}, \mathrm{N}$ \\
\hline Francisco et al. [108] & Portugal & CL $(n=2)$ & $\mathrm{CT}, \mathrm{CT}$ & $\mathrm{N}, \mathrm{N}$ \\
\hline Girard et al. [109] & Switzerland & $\mathrm{LC}$ and $\mathrm{OV}$ & $\mathrm{B}, \mathrm{B}$ & $\mathrm{N}, \mathrm{N}$ \\
\hline Guerreiro et al. [110] & Portugal & $\mathrm{CL}(n=4)$ & $\mathrm{CT}, \mathrm{CT}, \mathrm{CT}, \mathrm{CT}, \mathrm{CT}$ & $\mathrm{N}, \mathrm{N}, \mathrm{N}, \mathrm{N}, \mathrm{N}$ \\
\hline Gruffat et al. [111] & France & OV $(n=2)$ & $\mathrm{CT}, \mathrm{CT}$ & $\mathrm{N}, \mathrm{N}$ \\
\hline Hart et al. [112] & United Kingdom & Pisum sativum $(n=4)$ & $\mathrm{CT}, \mathrm{CT}, \mathrm{CT}, \mathrm{CT}, \mathrm{CT}$ & $\mathrm{N}, \mathrm{N}, \mathrm{N}, \mathrm{N}, \mathrm{N}$ \\
\hline Hassan et al. [113] & Egypt & Punica granatum, MI, B & B, B, B & $\mathrm{N}, \mathrm{N}, \mathrm{N}$ \\
\hline Hatami et al. [114] & Iran & Punica granatum $(n=3)$ & $\mathrm{B}, \mathrm{B}$ & $\mathrm{N}, \mathrm{N}$ \\
\hline Jerónimo et al. [115] & Portugal & $\operatorname{VV}(n=2)$, CL $(n=2)$ & $\mathrm{CT}, \mathrm{CT}, \mathrm{CT}, \mathrm{CT}, \mathrm{CT}$ & $\mathrm{E}, \mathrm{N}, \mathrm{E}, \mathrm{N}$ \\
\hline Jerónimo et al. [116] & Portugal & $\mathrm{VV}(n=2), \mathrm{CL}(n=2)$ & $\mathrm{CT}, \mathrm{CT}, \mathrm{CT}, \mathrm{CT}, \mathrm{CT}$ & $\mathrm{E}, \mathrm{N}, \mathrm{E}, \mathrm{N}$ \\
\hline Kamel et al. [117] & Saudi Arabia & QU $(n=2)$ & CT, CT & E, E \\
\hline Kazemi and Mokhtarpour [118] & Iran & Prunus amygdalus $(n=3)$ & $\mathrm{B}, \mathrm{B}, \mathrm{B}$ & $\mathrm{N}, \mathrm{N}, \mathrm{N}$ \\
\hline Leparmarai et al. [119] & Switzerland & VV & $\mathrm{B}$ & E \\
\hline Lima et al. [120] & Brazil & Macrotyloma axillare & B & $\mathrm{N}$ \\
\hline Liu et al. [21] & China & $\mathrm{CH}(n=2)$ & HT, HT & $\mathrm{E}, \mathrm{E}$ \\
\hline López-Andrés et al. [91] & Italy & QU & $\mathrm{CT}$ & $\mathrm{E}$ \\
\hline Majewska and Kowalik [121] & Poland & VAC, Quercus sp. & $\mathrm{B}, \mathrm{B}$ & $\mathrm{N}, \mathrm{N}$ \\
\hline Flores et al. [122] & Brazil & $\operatorname{VV}(n=3)$ & $\mathrm{B}, \mathrm{B}, \mathrm{B}$ & $\mathrm{N}, \mathrm{N}, \mathrm{N}$ \\
\hline Flores et al. [123] & Brazil & $\operatorname{VV}(n=3)$ & $\mathrm{B}, \mathrm{B}, \mathrm{B}$ & $\mathrm{N}, \mathrm{N}, \mathrm{N}$ \\
\hline Moghaddam et al. [124] & Iran & Berberis vulgaris $(n=2)$ & B, B & $\mathrm{N}, \mathrm{N}$ \\
\hline Natalello et al. [18] & Italy & Punica granatum & B & $\mathrm{N}$ \\
\hline Nobre et al. [125] & Brazil & Psidium guajava $(n=4)$ & $\mathrm{B}, \mathrm{B}, \mathrm{B}, \mathrm{B}, \mathrm{B}$ & $\mathrm{N}, \mathrm{N}, \mathrm{N}, \mathrm{N}, \mathrm{N}$ \\
\hline Norouzian and Ghiasi [126] & Iran & Pistacia vera $(n=3)$ & $\mathrm{B}, \mathrm{B}, \mathrm{B}$ & $\mathrm{N}, \mathrm{N}, \mathrm{N}$ \\
\hline Obeidat et al. [127] & Jordan & Ceratonia siliqua $(n=2)$ & CT, CT & $\mathrm{N}, \mathrm{N}$ \\
\hline Odhaib et al. [128] & Malaysia & $\mathrm{RO}(n=3), \mathrm{NS}(n=3), \mathrm{B}(n=3)$ & $\mathrm{B}(n=9)$ & $\mathrm{N}(n=9)$ \\
\hline Pathak et al. [13] & India & B, B & $\mathrm{CT}, \mathrm{CT}$ & $\mathrm{N}, \mathrm{N}$ \\
\hline Peng et al. [95] & Canada & Dalea purpurea & CT & $\mathrm{N}$ \\
\hline Po et al. [129] & Australia & Ilex paraguarensis & $\mathrm{CT}$ & $\mathrm{N}$ \\
\hline Priolo et al. [16] & Italy & Corylus avellana & $\mathrm{B}$ & $\mathrm{N}$ \\
\hline Rajabi et al. [130] & Iran & Punica granatum $(n=3)$ & $\mathrm{B}, \mathrm{B}, \mathrm{B}$ & $\mathrm{N}, \mathrm{N}, \mathrm{N}$ \\
\hline Rojas-Román et al. [15] & Mexico & B, B, B & $\mathrm{B}, \mathrm{B}, \mathrm{B}$ & E, E, E \\
\hline Sanchez et al. [131] & Mexico & Caesalpinia coriaria & $\mathrm{B}$ & $\mathrm{N}$ \\
\hline Sena et al. [132] & Brazil & Passiflora edulis $(n=3)$ & $\mathrm{CT}, \mathrm{CT}, \mathrm{CT}$ & $\mathrm{N}, \mathrm{N}, \mathrm{N}$ \\
\hline Sharifi and Chaji [133] & Iran & Punica granatum $(n=3)$ & $\mathrm{B}, \mathrm{B}, \mathrm{B}$ & $\mathrm{N}, \mathrm{N}, \mathrm{N}$ \\
\hline SoltaniNezhad et al. [134] & Iran & Pistacia vera $(n=3)$ & $\mathrm{B}, \mathrm{B}, \mathrm{B}$ & $\mathrm{N}, \mathrm{N}, \mathrm{N}$ \\
\hline Sun et al. [135] & China & Sorghum bicolor $(n=3)$ & $\mathrm{CT}, \mathrm{CT}, \mathrm{CT}$ & $\mathrm{N}, \mathrm{N}, \mathrm{N}$ \\
\hline Valenti et al. [17] & Italy & $\mathrm{AM}, \mathrm{QU}, \mathrm{CS}$ & CT, HT, HT & $\mathrm{E}, \mathrm{E}, \mathrm{E}$ \\
\hline Wang et al. [22] & Ireland & Corylus avellana $(n=2)$ & B, B & N, N \\
\hline Yisehak et al. [136] & Ethiopia & Albizia gummifera & CT & $\mathrm{N}$ \\
\hline Zhao et al. [137] & China & No reported & $\mathrm{B}, \mathrm{B}$ & $\mathrm{E}, \mathrm{E}$ \\
\hline Zhong et al. [19] & China & Sorghum bicolor $(n=3)$ & $\mathrm{CT}, \mathrm{CT}, \mathrm{CT}$ & $\mathrm{N}, \mathrm{N}, \mathrm{N}$ \\
\hline
\end{tabular}

B: blend; PA: Pimpinela anisum; ER: Eucalyptus rudis; AM: Acacia mearnsii; CS: Cesalpinia spinosa; CH: chestnut (Castanea sativa); QU: quebracho (Schinopsis spp.); VV: Vitis vinifera; CL: Cistus ladanifer; OP: Orbignya phalerata; Clep: Combretum leprosum; LC: Lotus corniculatus; OV: Onobrychis viciifolia; MI: Mangifera indica; VAC: Vaccinium vitis-ideae; sorghum (Sorghum bicolor); HT: hidrolisable tannin; CT: condensed tannin; E: extract; N: naturally present. $n$ : number of comparisons. 


\section{References}

1. Huang, Q.; Liu, X.; Zhao, G.; Hu, T.; Wang, Y. Potential and challenges of tannins as an alternative to in-feed antibiotics for farm animal production. Anim. Nutr. 2018, 4, 137-150. [CrossRef]

2. Mund, M.D.; Khan, U.H.; Tahir, U.; Mustafa, B.E.; Fayyaz, A. Antimicrobial drug residues in poultry products and implications on public health: A review. Int. J. Food Prop. 2017, 20, 1433-1446. [CrossRef]

3. Wang, H.; Ren, L.; Yu, X.; Hu, J.; Chen, Y.; He, G.; Jiang, Q. Antibiotic residues in meat, milk and aquatic products in Shanghai and human exposure assessment. Food Control 2017, 80, 217-225. [CrossRef]

4. Callaway, T.R.; Lillehoj, H.; Chuanchuen, R.; Gay, C.G. Alternatives to Antibiotics: A Symposium on the Challenges and Solutions for Animal Health and Production. Antibiotics 2021, 10, 471. [CrossRef]

5. Valenzuela-Grijalva, N.V.; Pinelli-Saavedra, A.; Muhlia-Almazan, A.; Domínguez-Díaz, D.; González-Ríos, H. Dietary inclusion effects of phytochemicals as growth promoters in animal production. J. Anim. Sci. Technol. 2017, 59, 8. [CrossRef] [PubMed]

6. Naumann, H.D.; Tedeschi, L.O.; Zeller, W.E.; Huntley, N.F. The role of condensed tannins in ruminant animal production: Advances, limitations and future directions. Rev. Bras. Zootec. 2017, 46, 929-949. [CrossRef]

7. Serra, V.; Salvatori, G.; Pastorelli, G. Dietary Polyphenol Supplementation in Food Producing Animals: Effects on the Quality of Derived Products. Animals 2021, 11, 401. [CrossRef]

8. Jerónimo, E.; Pinheiro, C.; Lamy, E.; Dentinho, M.T.; Sales-Baptista, E.; Lopes, O.; Capela e Silva, F. Tannins in ruminant nutrition: Impact on animal performance and quality of edible products. In Tannins: Biochemistry, Food Sources and Nutritional Properties; Combs, C.A., Ed.; Nova Science Publishers Inc.: New York, NY, USA, 2016; pp. 121-168.

9. Min, B.R.; Barry, T.N.; Attwood, G.T.; McNabb, W.C. The effect of condensed tannins on the nutrition and health of ruminants fed fresh temperate forages: A review. Anim. Feed Sci. Technol. 2003, 106, 3-19. [CrossRef]

10. Frutos, P.; Hervás, G.; Giráldez, F.J.; Mantecón, A.R. Review. Tannins and ruminant nutrition. Span. J. Agric. Res. 2004, 2, 191-202. [CrossRef]

11. Patra, A.K.; Saxena, J. Exploitation of dietary tannins to improve rumen metabolism and ruminant nutrition. J. Sci. Food Agric. 2011, 91, 24-37. [CrossRef]

12. Pimentel, P.R.S.; Pelelgrini, C.B.; Lanna, D.P.D.; Brant, L.M.S.; Ribeiro, C.V.D.M.; Silva, T.M.; Barbosa, A.M.; da Silva, J.J.M.; Bezerra, L.R.; Oliveira, R.L. Effects of Acacia mearnsii extract as a condensed-tannin source on animal performance, carcass yield and meat quality in goats. Anim. Feed Sci. Technol. 2021, 271, 114733. [CrossRef]

13. Pathak, A.K.; Dutta, N.; Pattanaik, A.K.; Chaturvedi, V.B.; Sharma, K. Effect of condensed tannins from Ficus infectoria and Psidium guajava leaf meal mixture on nutrient metabolism, methane emission and performance of lambs. Asian-Australas. J. Anim. Sci. 2017, 30, 1702-1710. [CrossRef]

14. Fernandes, J.; Pereira, F.J.; Menezes, D.; Caldas, A.C.; Cavalcante, I.; Oliveira, J.; Silva, J.J.; Cézar, M.; Bezerra, L. Carcass and meat quality in lambs receiving natural tannins from Mimosa tenuiflora hay. Small Rumin. Res. 2021, 198, 106362. [CrossRef]

15. Rojas-Román, L.; Castro-Pérez, B.; Estrada-Angulo, A.; Angulo-Montoya, C.; Yocupicio-Rocha, J.; López-Soto, M.; Barreras, A.; Zinn, R.A.; Plascencia, A. Influence of long-term supplementation of tannins on growth performance, dietary net energy and carcass characteristics: Finishing lambs. Small Rumin. Res. 2017, 153, 137-141. [CrossRef]

16. Priolo, A.; Valenti, B.; Natalello, A.; Bella, M.; Luciano, G.; Pauselli, M. Fatty acid metabolism in lambs fed hazelnut skin as a partial replacer of maize. Anim. Feed Sci. Technol. 2021, 272, 114794. [CrossRef]

17. Valenti, B.; Natalello, A.; Vasta, V.; Campidonico, L.; Roscini, V.; Mattioli, S.; Pauselli, M.; Priolo, A.; Lanza, M.; Luciano, G. Effect of different dietary tannin extracts on lamb growth performances and meat oxidative stability: Comparison between mimosa, chestnut and tara. Animal 2019, 13, 435-443. [CrossRef]

18. Natalello, A.; Priolo, A.; Valenti, B.; Codini, M.; Mattioli, S.; Pauselli, M.; Puccio, M.; Lanza, M.; Stergiadis, S.; Luciano, G. Dietary pomegranate by-product improves oxidative stability of lamb meat. Meat Sci. 2020, 162, 108037. [CrossRef]

19. Zhong, R.Z.; Fang, Y.; Wang, Y.; Sun, H.; Zhou, D. Effects of substituting finely ground sorghum for finely ground corn on feed digestion and meat quality in lambs infected with Haemonchus contortus. Anim. Feed Sci. Technol. 2015, 211, 31-40. [CrossRef]

20. Dentinho, M.T.P.; Paulos, K.; Francisco, A.; Belo, A.T.; Jerónimo, E.; Almeida, J.; Bessa, R.J.B.; Santos-Silva, J. Effect of soybean meal treatment with Cistus ladanifer condensed tannins in growth performance, carcass and meat quality of lambs. Livest. Sci. 2020, 236, 104021. [CrossRef]

21. Liu, H.; Li, K.; Lv, M.; Zhao, J.; Xiong, B. Effects of chestnut tannins on the meat quality, welfare, and antioxidant status of heat-stressed lambs. Meat Sci. 2016, 116, 236-242. [CrossRef]

22. Wang, S.; Giller, K.; Hillmann, E.; Marquardt, S.; Schwarm, A. Effect of supplementation of pelleted hazel (Corylus avellana) leaves on blood antioxidant activity, cellular immune response and heart beat parameters in sheep. J. Anim. Sci. 2019, 97, 4496-4502. [CrossRef] [PubMed]

23. Caprarulo, V.; Giromini, C.; Rossi, L. Review: Chestnut and quebracho tannins in pig nutrition: A review of the effects on performance and intestinal health. Animal 2021, 15, 100064. [CrossRef] [PubMed]

24. Manessis, G.; Kalogianni, A.I.; Lazou, T.; Moschovas, M.; Bossis, I.; Gelasakis, A.I. Plant-Derived Natural Antioxidants in Meat and Meat Products. Antioxidants 2020, 9, 1215. [CrossRef]

25. Sauvant, D.; Schmidely, P.; Daudin, J.J.; St-Pierre, N.R. Meta-analyses of experimental data in animal nutrition. Animal 2008, 2, 1203-1214. [CrossRef] [PubMed] 
26. Borenstein, M.; Hedges, L.V.; Higgins, J.P.T.; Rothstein, H.R. Introduction to Meta-Analysis, 1st ed.; John Wiley \& Sons: Chichester, UK, 2009; p. 413.

27. Doré, T.; Makowski, D.; Malézieux, E.; Munier-Jolain, N.; Tchamitchian, M.; Tittonell, P. Facing up to the paradigm of ecological intensification in agronomy: Revisiting methods, concepts and knowledge. Eur. J. Agron. 2011, 34, 197-210. [CrossRef]

28. Higgins, J.P.T.; Thompson, S.G.; Deeks, J.J.; Altman, D.G. Measuring inconsistency in meta-analysis. BMJ 2003, 327, 557-560. [CrossRef]

29. Abhijith, A.; Dunshea, F.R.; Warner, R.D.; Leury, B.J.; Ha, M.; Chauhan, S.S. A Meta-Analysis of the Effectiveness of High, Medium, and Low Voltage Electrical Stimulation on the Meat Quality of Small Ruminants. Foods 2020, 9, 1587. [CrossRef]

30. Moher, D.; Liberati, A.; Tetzlaff, J.; Altman, D.G.; Prisma Group. Preferred Reporting Items for Systematic Reviews and Meta-Analyses: The PRISMA Statement. PLoS Med. 2009, 6, e1000097. [CrossRef] [PubMed]

31. Herremans, S.; Vanwindekens, F.; Decruyenaere, V.; Beckers, Y.; Froidmont, E. Effect of dietary tannins on milk yield and composition, nitrogen partitioning and nitrogen use efficiency of lactating dairy cows: A meta-analysis. J. Anim. Physiol. Anim. Nutr. 2020, 104, 1209-1218. [CrossRef]

32. Orzuna-Orzuna, J.F.; Dorantes-Iturbide, G.; Lara-Bueno, A.; Mendoza-Martínez, G.D.; Miranda-Romero, L.A.; Hernández-García, P.A. Effects of Dietary Tannins' Supplementation on Growth Performance, Rumen Fermentation, and Enteric Methane Emissions in Beef Cattle: A Meta-Analysis. Sustainability 2021, 13, 7410. [CrossRef]

33. Lean, I.J.; Thompson, J.M.; Dunshea, F.R. A Meta-Analysis of Zilpaterol and Ractopamine Effects on Feedlot Performance, Carcass Traits and Shear Strength of Meat in Cattle. PLoS ONE 2014, 9, e115904. [CrossRef] [PubMed]

34. Belanche, A.; Newbold, C.J.; Morgavi, D.P.; Bach, A.; Zweifel, B.; Yáñez-Ruiz, D.R. A Meta-analysis Describing the Effects of the Essential oils Blend Agolin Ruminant on Performance, Rumen Fermentation and Methane Emissions in Dairy Cows. Animals 2020, 10, 620. [CrossRef] [PubMed]

35. Higgins, J.; Thomas, J. Cochrane Handbook for Systematic Reviews of Interventions, 2nd ed.; John Wiley and Sons Ltd: Chichester, UK, 2019; pp. 143-176.

36. Wallace, B.C.; Lajeunesse, M.J.; Dietz, G.; Dahabreh, I.J.; Trikalinos, T.A.; Schmid, C.H.; Gurevitch, J. OpenMEE: Intuitive, open-source software for metaanalysis in ecology and evolutionary biology. Methods Ecol. Evol. 2016, 8, 941-947. [CrossRef]

37. Hedges, L.V. Distribution theory for Glass's estimator of effect size and related estimators. J. Educ. Stat. 1981, 6, 107-128. [CrossRef]

38. Der Simonian, R.; Laird, N. Meta-analysis in clinical trials. Control. Clin. Trials 1986, 7, 177-188. [CrossRef]

39. Lean, I.J.; Rabiee, A.R.; Duffield, T.F.; Dohoo, I.R. Invited review: Use of meta-analysis in animal health and reproduction: Methods and applications. J. Dairy Sci. 2009, 92, 3545-3565. [CrossRef] [PubMed]

40. SAS (Statistical Analysis System). SAS/STAT User's Guide (Release 6.4); SAS Inst.: Cary, NC, USA, 2017.

41. Torres, R.N.S.; Moura, D.C.; Ghedini, C.P.; Ezequiel, J.M.B.; Almeida, M.T.C. Meta-analysis of the effects of essential oils on ruminal fermentation and performance of sheep. Small Rumin. Res. 2020, 189, 106148. [CrossRef]

42. Higgins, J.P.T.; Thompson, S.G. Quantifying heterogeneity in a meta-analysis. Stat. Med. 2002, 21, 1539-1558. [CrossRef] [PubMed]

43. Egger, M.; Smith, G.D.; Altman, D.G. Systematic Reviews in Health Care, 2nd ed.; MBJ Publishing Group: London, UK, 2001; pp. 109-121.

44. Littell, J.H.; Corcoran, J.; Pillai, V. Systematic Reviews and Meta-Analysis, 1st ed.; Oxford University Press: Oxford, UK, 2008; pp. 111-132.

45. Sterne, J.A.C.; Harbord, R.M. Funnel plots in meta-analysis. Stata J. 2004, 4, 127-141. [CrossRef]

46. Begg, C.B.; Mazumdar, M. Operating Characteristics of a Rank Correlation Test for Publication Bias. Biometrics 1994, 50, 1088-1101. [CrossRef]

47. Egger, M.; Smith, G.D.; Schneider, M.; Minder, C. Bias in meta-analysis detected by a simple, graphical test. BMJ 1997, 315, 629-634. [CrossRef]

48. Ioannidis, J.P.A.; Trikalinos, T.A. The appropriateness of asymmetry tests for publication bias in meta-analyses: A large survey. CMAJ 2007, 176, 1091-1096. [CrossRef]

49. Duval, S.; Tweedie, R. A nonparametric "trim and fill" method of accounting for publication bias in meta-analysis. J. Amer. Statist. Assoc. 2000, 95, 89-98. [CrossRef]

50. Toldrá, F. Lawrie's Meat Science, 8th ed.; Woodhead Publishing Limited: Cambridge, UK, 2017; 713p.

51. Orlandi, T.; Kozloski, G.V.; Alves, T.P.; Mesquita, F.R.; Ávila, S.C. Digestibility, ruminal fermentation and duodenal flux of amino acids in steers fed grass forage plus concentrate containing increasing levels of Acacia mearnsii tannin extract. Anim. Feed Sci. Technol. 2015, 210, 37-45. [CrossRef]

52. Yang, K.; Wei, C.; Zhao, G.; Xu, Z.; Lin, S. Dietary supplementation of tannic acid modulates nitrogen excretion pattern and urinary nitrogenous constituents of beef cattle. Livest. Sci. 2016, 191, 148-152. [CrossRef]

53. Bhattacharyya, A.; Chattopadhyay, R.; Mitra, S.; Crowe, S.E. Oxidative Stress: An Essential Factor in the Pathogenesis of Gastrointestinal Mucosal Diseases. Physiol. Rev. 2014, 94, 329-354. [CrossRef] [PubMed]

54. Wang, Y.; Chen, Y.; Zhang, X.; Lu, Y.; Chen, H. New insights in intestinal oxidative stress damage and the health intervention effects of nutrients: A review. J. Funct. Foods 2020, 75, 104248. [CrossRef] 
55. Dong, S.; Li, H.; Gasco, L.; Xiong, Y.; Guo, K.J.; Zoccarato, I. Antioxidative activity of the polyphenols from the involucres of Castanea mollissima Blume and their mitigating effects on heat stress. Poult. Sci. 2015, 94, 1096-1104. [CrossRef] [PubMed]

56. Gessner, D.K.; Ringseis, R.; Eder, K. Potential of Plant Polyphenols to Combat Oxidative Stress and Inflammatory Processes in Farm Animals. J. Anim. Physiol. Anim. Nutr. 2017, 101, 605-628. [CrossRef] [PubMed]

57. Tedeschi, L.O.; Muir, J.P.; Naumann, H.D.; Norris, A.B.; Ramirez-Restrepo, C.A.; Mertens-Talcott, S.U. Nutritional Aspects of Ecologically Relevant Phytochemicals in Ruminant Production. Front. Vet. Sci. 2021, 8, 628445. [CrossRef]

58. Lesschaeve, I.; Noble, A.C. Polyphenols: Factors influencing their sensory properties and their effects on food and beverage preferences. Am. J. Clin. Nutr. 2005, 81, 330S-335S. [CrossRef]

59. Glendinning, J.I. Is the bitter rejection response always adaptive? Physiol. Behav. 1994, 56, 1217-1227. [CrossRef]

60. Lamy, E.; Da Costa, G.; Santos, R.; Capela e Silva, F.; Potes, J.; Pereira, A.; Coelho, A.V.; Baptista, E.S. Effect of condensed tannin ingestion in sheep and goat parotid saliva proteome. J. Anim. Physiol. Anim. Nutr. 2011, 95, 304-312. [CrossRef]

61. Lamy, E.; Rodrigues, L.; Guerreiro, O.; Soldado, D.; Francisco, A.; Lima, M.; Silva, F.C.E.; Lopes, O.; Santos-Silva, J.; Jerónimo, E. Changes in salivary protein composition of lambs supplemented with aerial parts and condensed tannins: Extract from Cistus ladanifer L.-A preliminary study. Agrofor. Syst. 2020, 94, 1501-1559. [CrossRef]

62. Austin, P.J.; Suchar, L.A.; Robbins, C.T.; Hagerman, A.E. Tannin-binding proteins in saliva of deer and their absence in saliva of sheep and cattle. J. Chem. Ecol. 1989, 15, 1335-1347. [CrossRef]

63. McArthur, C.; Sanson, G.D.; Beal, A.M. Salivary proline-rich proteins in mammals-Roles in oral homeostasis and counteracting dietary tannin. J. Chem. Ecol. 1995, 21, 663-691. [CrossRef] [PubMed]

64. Lamy, E.; Rawel, H.; Schweigert, F.J.; Capela e Silva, F.; Ferreira, A.; Costa, A.R.; Antunes, C.; Almeida, A.M.; Coelho, A.V.; Sales-Baptista, E. The Effect of Tannins on Mediterranean Ruminant Ingestive Behavior: The Role of the Oral Cavity. Molecules 2011, 16, 2766-2784. [CrossRef] [PubMed]

65. Corazzin, M.; Del Bianco, S.; Bovolenta, S.; Piasentier, E. Carcass Characteristics and Meat Quality of Sheep and Goat. In More than Beef, Pork and Chicken-The Production, Processing, and Quality Traits of Other Sources of Meat for Human Diet; Lorenzo, J.M., Munekata, P.E.S., Barba, F., Toldrá, F., Eds.; Springer International Publishing: Cham, Switzerland, 2019; pp. 119-165. ISBN 978-3-030-05483-0.

66. Liang, H.; Xu, L.; Zhao, X.; Pan, K.; Yi, Z.; Bai, J.; Qi, X.; Xin, J.; Li, M.; Ouyang, K.; et al. RNA-Seq analysis reveals the potential molecular mechanisms of daidzein on adipogenesis in subcutaneous adipose tissue of finishing Xianan beef cattle. J. Anim. Physiol. Anim. Nutr. 2020, 104, 1-11. [CrossRef] [PubMed]

67. Qin, X.; Zhang, T.; Cao, Y.; Deng, B.; Zhang, J.; Zhao, J. Effects of dietary sea buckthorn pomace supplementation on skeletal muscle mass and meat quality in lambs. Meat Sci. 2020, 166, 108141. [CrossRef]

68. Biondi, L.; Rabdazzo, C.L.; Russo, N.; Pino, A.; Natalello, A.; Van Hoorde, K.; Caggia, C. Dietary Supplementation of TanninExtracts to Lambs: Effects on Meat Fatty Acids Composition and Stability and on Microbial Characteristics. Foods $2019,8,469$. [CrossRef]

69. Cimmino, R.; Barone, C.M.A.; Claps, S.; Varricchio, E.; Rufrano, D.; Caroprese, M.; Albenzio, M.; De Palo, P.; Campanile, G.; Neglia, G. Effects of dietary supplementation with polyphenols on meat quality in Saanen goat kids. BMC Vet. Res. 2018, 14, 181. [CrossRef]

70. Calnan, H.; Jacob, R.; Pethick, D.; Gardner, G. Factors affecting the colour of lamb meat from the longissimus muscle during display: The influence of muscle weight and muscle oxidative capacity. Meat Sci. 2014, 96, 1049-1057. [CrossRef]

71. Komprda, T.; Kuchtík, J.; Jarošová, A.; Dračková, E.; Zemánek, L.; Filipčík, R. Meat quality characteristics of lambs of three organically raised breeds. Meat Sci. 2012, 91, 499-505. [CrossRef] [PubMed]

72. Teixeira, A.; Jimenez-Badillo, M.R.; Rodriguez, S. Effect of sex and carcass weight on carcass traits and meat quality in goat kids of cabrito transmontano. Span. J. Agric. Res. 2011, 9, 753-760. [CrossRef]

73. Fruet, A.P.B.; Giotto, F.M.; Fonseca, M.A.; Nörnberg, J.L.; de Mello, A.S. Effects of the incorporation of tannin extract from quebracho colorado wood on color parameters, lipid oxidation, and sensory attributes of beef patties. Foods 2020, 9, 667. [CrossRef] [PubMed]

74. Contini, C.; Alvarez, R.; O'Sullivan, M.; Dowling, D.P.; Gargan, S.O.; Monahan, F.J. Effect of an active packaging with citrus extract on lipid oxidation and sensory quality of cooked turkey meat. Meat Sci. 2014, 96, 1171-1176. [CrossRef]

75. Fraga-Corral, M.; Otero, P.; Echave, J.; GarciaOliveira, P.; Carpena, M.; Jarboui, A.; Nuñez-Estevez, B.; Simal-Gandara, J.; Prieto, M.A. By-Products of Agri-Food Industry as Tannin-Rich Sources: A Review of Tannins' Biological Activities and Their Potential for Valorization. Foods 2021, 10, 137. [CrossRef]

76. Malheiros, J.M.; Braga, C.P.; Grove, R.A.; Ribeiro, F.A.; Calkins, C.R.; Adamec, J.; Chardulo, L.A.L. Influence of oxidative damage to proteins on meat tenderness using a proteomics approach. Meat Sci. 2019, 148, 64-71. [CrossRef] [PubMed]

77. Zhong, R.Z.; Zhou, D.W.; Tan, C.Y.; Tan, Z.L.; Han, X.F.; Zhou, C.S.; Tang, S.X. Effect of tea catechins on regulation of antioxidant enzyme expression in $\mathrm{H}_{2} \mathrm{O}_{2}$-induced skeletal muscle cells of goat in vitro. J. Agric. Food Chem. 2011, 59, 11338-11343. [CrossRef]

78. Piccione, G.; Casella, S.; Giannetto, C.; Bazzano, M.; Giudice, E.; Fazio, F. Oxidative stress associated with road transportation in ewes. Small Rumin. Res. 2013, 112, 235-238. [CrossRef]

79. Ablikim, B.; Liu, Y.; Kerim, A.; Shen, P.; Abdurerim, P.; Zhou, G.H. Effects of breed, muscle type, and frozen storage on physico-chemical characteristics of lamb meat and its relationship with tenderness. CyTA J. Food 2016, 14, 109-116. [CrossRef] 
80. Hocquette, J.F.; Gondret, F.; Baéza, E.; Médale, F.; Jurie, C.; Pethick, D.W. Intramuscular fat content in meat-producing animals: Development, genetic and nutritional control, and identification of putative markers. Animal 2010, 4, 303-319. [CrossRef] [PubMed]

81. Jin, Q.; Liu, G.; Tan, X.; Zhang, X.; Liu, X.; Wei, C. Gallic acid as a key substance to inhibit proliferation and adipogenesis in bovine subcutaneous adipocyte. Anim. Biotechnol. 2020, 1-7. [CrossRef]

82. Lin, J.; Della-Fera, M.A.; Baile, C.A. Green tea polyphenol epigallocatechin gallate inhibits adipogenesis and induces apoptosis in 3T3-L1 adipocytes. Obes. Res. 2005, 13, 982-990. [CrossRef]

83. Liu, X.; Kim, J.K.; Li, Y.; Li, J.; Liu, F.; Chen, X. Tannic acid stimulates glucose transport and inhibits adipocyte differentiation in 3T3-L1 cells. J. Nutr. 2005, 135, 165-171. [CrossRef]

84. Barlocco, N.; Vadell, A.; Ballesteros, F.; Galietta, G.; Cozzolino, D. Predicting intramuscular fat, moisture and Warner-Bratzler shear force in pork muscle using near infrared reflectance spectroscopy. Anim. Sci. 2006, 82, 111-116. [CrossRef]

85. Pateiro, M.; Barba, F.J.; Domínguez, R.; Sant'Ana, A.S.; Mousavi Khaneghah, A.; Gavahian, M.; Gómez, B.; Lorenzo, J.M. Essential oils as natural additives to prevent oxidation reactions in meat and meat products: A review. Food Res. Int. 2018, 113, 156-166. [CrossRef] [PubMed]

86. Faustman, C.; Sun, Q.; Mancini, R.; Suman, S. Myoglobin and lipid oxidation interactions: Mechanistic bases and control. Meat Sci. 2010, 86, 86-94. [CrossRef]

87. Liu, H.; Zhou, D.; Li, K. Effects of chestnut tannins on performance and antioxidative status of transition dairy cows. J. Dairy Sci. 2013, 96, 5901-5907. [CrossRef]

88. Chivandi, E.; Dangarembizi, R.; Nyakudya, T.T.; Erlwanger, K.H. Use of Essential Oils as a Preservative of Meat. In Essential Oils in Food Preservation, Flavor and Safety; Elsevier: Amsterdam, The Netherlands, 2016. [CrossRef]

89. Jami, E.; Israel, A.; Kotser, A.; Mizrahi, I. Exploring the bovine rumen bacterial community from birth to adulthood. ISME J. 2013, 7, 1069-1079. [CrossRef]

90. Gladine, C.; Rock, E.; Morand, C.; Bauchart, D.; Durand, D. Bioavailability and Antioxidant Capacity of Plant Extracts Rich in Polyphenols, given as a Single Acute Dose, in Sheep Made Highly Susceptible to Lipoperoxidation. Br. J. Nutr. 2007, 98, 691-701. [CrossRef] [PubMed]

91. López-Andrés, P.; Luciano, G.; Vasta, V.; Gibson, T.M.; Biondi, L.; Priolo, A.; Mueller-Harvey, I. Dietary quebracho tannins are not absorbed, but increase the antioxidant capacity of liver and plasma in sheep. Br. J. Nutr. 2013, 110, 632-639. [CrossRef] [PubMed]

92. Ghiselli, A.; Serafini, M.; Natella, F.; Scaccini, C. Total antioxidant capacity as a tool to assess redox status: Critical view and experimental data. Free Radic. Biol. Med. 2000, 29, 1106-1114. [CrossRef]

93. Celi, P. Oxidative stress in ruminants. In Studies on Veterinary Medicine. Oxidative Stress in Applied Basic Research and Clinical Practice; Mandelker, L., Vajdovich, P., Eds.; Humana Press: Totowa, NJ, USA; New York, NY, USA, 2011 ; pp. $191-231$. ISBN 978-1-61779-070-6.

94. Cuervo, W.; Sordillo, L.M.; Abuelo, A. Oxidative Stress Compromises Lymphocyte Function in Neonatal Dairy Calves. Antioxidants 2021, 10, 255. [CrossRef] [PubMed]

95. Peng, K.; Shirley, D.C.; Xu, Z.; Huang, Q.; Mcallister, T.A.; Chaves, A.V.; Acharya, S.; Liu, C.L.; Wang, S.X.; Wang, Y.X. Effect of purple prairie clover (Dalea purpurea vent.) hay and its condensed tannins on growth performance, wool growth, nutrient digestibility, blood metabolites and ruminal fermentation in lambs fed total mixed rations. Anim. Feed Sci. Technol. 2016, 222, 100-110. [CrossRef]

96. Masella, R.; Di Benedetto, R.; Varì, R.; Filesi, C.; Giovannini, C. Novel mechanisms of natural antioxidant compounds in biological systems: Involvement of glutathione and glutathione-related enzymes. J. Nutr. Biochem. 2005, 16, 577-586. [CrossRef]

97. Celi, P. Biomarkers of oxidative stress in ruminant medicine. Immunopharmacol. Immunotoxicol. 2011, 33, 233-240. [CrossRef]

98. Awawdeh, M.S.; Dager, H.K.; Obeidat, B.S. Effects of alternative feedstuffs on growth performance, carcass characteristics, and meat quality of growing Awassi lambs. Ital. J. Anim. Sci. 2019, 18, 777-785. [CrossRef]

99. Bandeira, P.A.V.; Pereira Filho, J.M.; Silva, A.M.A.; Cezar, M.F.; Bakke, A.O.; Silva, U.L.; Borburema, J.B.; Bezerra, L.R. Performance and carcass characteristics of lambs fed diets with increasing levels of Mimosa tenuiflora (Willd.) hay replacing Buffel grass hay. Trop. Anim. Health Prod. 2017, 49, 1001-1007. [CrossRef]

100. Bhatt, R.S.; Sahoo, A.; Sarkar, S.; Soni, L.; Sharma, P.; Gadekar, Y.P. Dietary supplementation of plant bioactive-enriched aniseed straw and eucalyptus leaves modulates tissue fatty acid profile and nuggets quality of lambs. Animal 2020, 14, $2642-2651$. [CrossRef]

101. Bonanno, A.; Di Miceli, G.; Di Grigoli, A.; Frenda, A.S.; Tornambè, G.; Giambalvo, D.; Amato, G. Effects of feeding green forage of sulla (Hedysarum coronarium L.) on lamb growth, gastrointestinal nematode infection, and carcass and meat quality. Animal 2011, 5, 148-154. [CrossRef]

102. Buccioni, A.; Pauselli, M.; Minieri, S.; Roscini, V.; Mannelli, F.; Rapaccini, S.; Lupi, P.; Conte, G.; Serra, A.; Cappucci, A. Chestnut or quebracho tannins in the diet of grazing ewes supplemented with soybean oil: Effects on animal performances, blood parameters and fatty acid composition of plasma and milk lipids. Small Rumin. Res. 2017, 153, 23-30. [CrossRef]

103. Chikwanha, O.C.; Moelich, E.; Gouws, P.; Muchenje, V.; Nolte, J.V.E.; Dugan, M.E.R.; Mapiye, C. Effects of feeding increasing levels of grape (Vitis vinifera cv. Pinotage) pomace on lamb shelf-life and eating quality. Meat Sci. 2019, 157, 107887. [CrossRef] [PubMed] 
104. Chikwanha, O.C.; Muchenje, V.; Nolte, J.E.; Dugan, M.E.R.; Mapiye, C. Grape pomace (Vitis vinifera L. cv. Pinotage) supplementation in lamb diets: Effects on growth performance, carcass and meat quality. Meat Sci. 2019, 147, 6-12. [CrossRef]

105. Costa, E.d.S.; Ribiero, C.; Silva, T.; Ribeiro, R.; Vieira, J.; Lima, A.d.O.; Barbosa, A.; da Silva, J.J.M.; Bezerra, L.R.; Oliveira, R.L. Intake, nutrient digestibility, nitrogen balance, serum metabolites and growth performance of lambs supplemented with Acacia mearnsii condensed tannin extract. Anim. Feed Sci. Technol. 2021, 272, 114744. [CrossRef]

106. Dey, A.; Dutta, N.; Pattanaik, A.K.; Sharma, K. Antioxidant status, metabolic profile and immune response of lambs supplemented with tannin rich Ficus infectoria leaf meal. Jpn. J. Vet. Res. 2015, 63, 15-24. [CrossRef]

107. Abdalla Filho, A.L.; Corrêa, P.S.; Lemos, L.N.; Dineshkumar, D.; Issakowicz, J.; Ieda, E.H.; Lima, P.M.T.; Barreal, M.; McManus, C.; Mui, T.S.; et al. Diets based on plants from Brazilian Caatinga altering ruminal parameters, microbial community and meat fatty acids of Santa Inês lambs. Small Rumin. Res. 2017, 154, 70-77. [CrossRef]

108. Francisco, A.; Alves, S.P.; Portugal, P.V.; Dentinho, M.T.; Jerónimo, E.; Sengo, S.; Almeida, J.; Bressan, M.C.; Pires, V.M.R.; Alfaia, C.M.; et al. Effects of dietary inclusion of citrus pulp and rockrose soft stems and leaves on meat quality and fatty acid composition. Animal 2017, 12, 872-881. [CrossRef]

109. Girard, M.; Dohme-Meier, F.; Silacci, P.; Ampuero Kragten, S.; Kreuzer, M.; Bee, G. Forage legumes rich in condensed tannins may increase n-3 fatty acid levels and sensory quality of lamb meat. J. Sci. Food Agric. 2016, 96, 1923-1933. [CrossRef]

110. Guerreiro, O.; Alves, S.P.; Soldado, D.; Cachucho, L.; Almeida, J.M.; Francisco, A.; Jerónimo, E. Inclusion of the aerial part and condensed tannin extract from Cistus ladanifer L. in lamb diets-Effects on growth performance, carcass and meat quality and fatty acid composition of intramuscular and subcutaneous fat. Meat Sci. 2020, 160, 107945. [CrossRef]

111. Gruffat, D.; Durand, D.; Rivaroli, D.; do Prado, I.N.; Prache, S. Comparison of muscle fatty acid composition and lipid stability in lambs stall-fed or pasture-fed alfalfa with or without sainfoin pellet supplementation. Animal 2020, 14, 1093-1101. [CrossRef]

112. Hart, K.J.; Sinclair, L.A.; Wilkinson, R.G.; Huntington, J.A. Effect of Whole-Crop Pea (Pisum Sativum L.) Silages Differing in Condensed Tannin Content as a Substitute for Grass Silage and Soybean Meal on the Performance, Metabolism, and Carcass Characteristics of Lambs. J. Anim. Sci. 2011, 89, 3663-3676. [CrossRef]

113. Hassan, T.M.M.; Ahmed-Farid, O.A.; Abdel-Fattah, F.A.I. Effects of different sources and levels of tannins on live performance and antioxidant response of Ossimi lambs. J. Agric. Sci. 2020, 158, 339-348. [CrossRef]

114. Hatami, A.; Alipour, D.; Hozhabri, F.; Tabatabaei, M. Effect of different levels of pomegranate marc with or without polyethylene glycol on performance, nutrients digestibility and protozoal population in growing lambs. Anim. Feed Sci. Tech. 2018, 235, 15-22. [CrossRef]

115. Jerónimo, E.; Alves, S.P.; Dentinho, M.T.P.; Martins, S.V.; Prates, J.A.M.; Vasta, V.; Santos-Silva, J.; Bessa, R.J.B. Effect of grape seed extract, Cistus ladanifer L. and vegetable oil supplementation on fatty acid composition of abomasal digesta and intramuscular fat of lambs. J. Agric. Food Chem. 2010, 58, 10710-10721. [CrossRef] [PubMed]

116. Jerónimo, E.; Alfaia, C.M.M.; Alves, S.P.; Dentinho, M.T.P.; Prates, J.A.M.; Vasta, V.; Santos-Silva, J.; Bessa, R.J.B. Effect of dietary grape seed extract and Cistus ladanifer L. in combination with vegetable oil supplementation on lamb meat quality. Meat Sci. 2012, 92, 841-847. [CrossRef] [PubMed]

117. Kamel, H.E.M.; Al-Dobaib, S.N.; Salem, A.Z.; Lopez, S.; Alaba, P.A. Influence of dietary supplementation with sunflower oil and quebracho tannins on growth performance and meat fatty acid profile of Awassi lambs. Anim. Feed Sci. Technol. 2018, 235, 97-104. [CrossRef]

118. Kazemi, M.; Mokhtarpour, A. In vitro and in vivo evaluation of some tree leaves as forage sources in the diet of Baluchi male lambs. Small Rumin. Res. 2021, 201, 106416. [CrossRef]

119. Leparmarai, P.T.; Sinz, S.; Kunz, C.; Liesegang, A.; Ortmann, S.; Kreuzer, M.; Marquardt, S. Transfer of total phenols from a grapeseed-supplemented diet to dairy sheep and goat milk, and effects on performance and milk quality. J. Anim. Sci. 2019, 97, 1840-1851. [CrossRef]

120. Lima, P.M.T.; Filho, A.L.A.; Issakowicz, J.; Ieda, E.H.; Corrêa, P.S.; Mattos, W.T.; Gerdes, L.; McManus, C.; Abdalla, A.L.; Louvandini, H. Methane emission, ruminal fermentation parameters and fatty acid profile of meat in Santa Inês lambs fed the legume macrotiloma. Anim. Prod. Sci. 2020, 60, 665-673. [CrossRef]

121. Majewska, M.P.; Kowalik, B. Growth Performance, Carcass Characteristics, Fatty Acid Composition, and Blood Biochemical Parameters of Lamb Fed Diet with the Addition of Lingonberry Leaves and Oak Bark. Eur. J. Lipid Sci. Technol. 2020, 122, 1900273. [CrossRef]

122. Flores, D.R.M.; da Fonseca, P.A.F.; Schmitt, J.; Tonetto, C.J.; Junior, A.G.R.; Hammerschmitt, R.K.; Facco, D.B.; Brunetto, G.; Nörnberg, J.L. Lambs fed with increasing levels of grape pomace silage: Effects on productive performance, carcass characteristics, and blood parameters. Livest. Sci. 2020, 240, 104169. [CrossRef]

123. Flores, D.R.M.; da Fonseca, P.A.F.; Schmitt, J.; Tonetto, C.J.; Junior, A.G.R.; Hammerschmitt, R.K.; Facco, D.B.; Brunetto, G.; Nörnberg, J.L. Lambs fed with increasing levels of grape pomace silage: Effects on meat quality. Small Rumin. Res. 2021, 195, 106234. [CrossRef]

124. Moghaddam, V.K.; Elahi, M.Y.; Nasri, M.H.F.; Elghandour, M.M.M.Y.; Monroy, J.C.; Salem, A.Z.M.; Karami, M.; Mlambo, V. Growth performance and carcass characteristics of finishing male lambs fed barberry pomace-containing diets. Anim. Biotechnol. 2019, 32, 178-184. [CrossRef] 
125. Nobre, P.T.; Munekata, P.E.; Costa, R.G.; Carvalho, F.R.; Ribeiro, N.L.; Queiroga, R.C.; Sousa, S.; Silva, A.C.R.; Lorenzo, J.M. The impact of dietary supplementation with guava (Psidium guajava L.) agroindustrial waste on growth performance and meat quality of lambs. Meat Sci. 2020, 164, 108105. [CrossRef] [PubMed]

126. Norouzian, M.A.; Ghiasi, S.E. Carcass performance and mineral content in Balouchi lamb fed pistachio byproduct. Meat Sci. 2012, 92, 157-159. [CrossRef]

127. Obeidat, B.S.; Alrababah, M.A.; Abdullah, A.Y.; Alhamad, M.N.; Gharaibeh, M.A.; Rababah, T.M.; Ishmais, M.A. Growth performance and carcass characteristics of Awassi lambs fed diets containing carob pods (Ceratonia siliqua L.). Small Rumin. Res. 2011, 96, 149-154. [CrossRef]

128. Odhaib, K.J.; Adeyemi, K.D.; Sazili, A.Q. Carcass traits, fatty acid composition, gene expression, oxidative stability and quality attributes of different muscles in Dorper lambs fed Nigella sativa seeds, Rosmarinus officinalis leaves and their combination. Asian-Australas. J. Anim. Sci. 2018, 31, 1345-1357. [CrossRef]

129. Po, E.; Horsburgh, K.; Raadsma, H.W.; Celi, P. Yerba Mate (Ilex paraguarensis) as a novel feed supplement for growing lambs. Small Rumin. Res. 2012, 106, 131-136. [CrossRef]

130. Rajabi, M.; Rouzbehan, Y.; Rezaei, J. A strategy to improve nitrogen utilization, reduce environmental impact, and increase performance and antioxidant capacity of fattening lambs using pomegranate peel extract. J. Anim. Sci. 2017, 95, 499-510. [CrossRef]

131. Sánchez, N.; Mendoza, G.D.; Martínez, J.A.; Hernández, P.A.; Camacho, L.M.; Lee-Rangel, H.A.; Vázquez, A.; Flores, R. Effect of Caesalpinia coriaria fruits and soybean oil on finishing lamb performance and meat characteristics. Biomed Res. Int. 2018, 1-6. [CrossRef] [PubMed]

132. Sena, J.A.B.; Villela, S.D.J.; Santos, R.A.; Pereira, I.G.; Castro, G.H.F.; Mourthe, M.H.F.; Bonfá, C.S.; Martins, P.G.M.A. Intake, digestibility, performance, and carcass traits of rams provided with dehydrated passion fruit (Passiflora edulis $\mathrm{f}$. flavicarpa) peel, as a substitute of Tifton 85 (Cynodon spp.). Small Rumin. Res. 2015, 129, 18-24. [CrossRef]

133. Sharifi, A.; Chaji, M. Effects of processed recycled poultry bedding with tannins extracted from pomegranate peel on the nutrient digestibility and growth performance of lambs. S. Afr. J. Anim. Sci. 2019, 49, 290-300. [CrossRef]

134. SoltaniNezhad, B.; Dayani, O.; Khezri, A.; Tahmasbi, R. Performance and carcass characteristics in fattening lambs fed diets with different levels of pistachio byproducts silage with wasted date. Small Rumin. Res. 2016, 137, 177-182. [CrossRef]

135. Sun, H.X.; Gao, T.S.; Zhong, R.Z.; Fang, Y.; Di, G.L.; Zhou, D.W. Effects of corn replacement by sorghum in diets on performance, nutrient utilization, blood parameters, antioxidant status and meat color stability in lambs. Can. J. Anim. Sci. 2018, 98, 723-731. [CrossRef]

136. Yisehak, K.; Biruk, K.; Abegaze, B.; Janssens, G.P.J. Growth of sheep fed tanninrich Albizia gummifera with or without polyethylene glycol. Trop. Anim. Health Prod. 2014, 46, 1113-1118. [CrossRef]

137. Zhao, M.D.; Di, L.F.; Tang, Z.Y.; Jiang, W.; Li, C.Y. Effect of tannins and cellulase on growth performance, nutrients digestibility, blood profiles, intestinal morphology and carcass characteristics in Hu sheep. Asian-Australas. J. Anim. Sci. 2019, 32, 1540-1547. [CrossRef] 